\title{
THE SEGAL CONJECTURE FOR TOPOLOGICAL HOCHSCHILD HOMOLOGY OF COMPLEX COBORDISM
}

\author{
SVERRE LUNØE-NIELSEN AND JOHN ROGNES
}

\begin{abstract}
We study the $C_{p}$-equivariant Tate construction on the topological Hochschild homology $\operatorname{THH}(B)$ of a symmetric ring spectrum $B$ by relating it to a topological version $R_{+}(B)$ of the Singer construction, extended by a natural circle action. This enables us to prove that the fixed and homotopy fixed point spectra of $\operatorname{THH}(B)$ are $p$-adically equivalent for $B=M U$ and $B P$. This generalizes the classical $C_{p}$-equivariant Segal conjecture, which corresponds to the case $B=S$.
\end{abstract}

\section{Contents}

1. Introduction

2. Strategy of proof

3. Equivariant approximations

4. Tate representatives

5. Cyclotomic structure

6. The comparison map

7. The Segal conjecture

References

\section{INTRODUCTION}

Let $p$ be a prime, let $\mathbb{T}$ be the circle group, and let $C_{p} \subset \mathbb{T}$ be the cyclic subgroup of order $p$. Let $B$ be any symmetric ring spectrum, in the sense of [20. Its topological Hochschild homology $\operatorname{THH}(B)$, as constructed in [19], is then a genuinely $\mathbb{T}$-equivariant spectrum in the sense of [21]. Let $E \mathbb{T}$ be a free, contractible $\mathbb{T}-\mathrm{CW}$ complex and let $c: E \mathbb{T}_{+} \rightarrow S^{0}$ be the usual collapse map. Forming the induced map $\operatorname{THH}(B)=F\left(S^{0}, \mathrm{THH}(B)\right) \rightarrow F\left(E \mathbb{T}_{+}, \mathrm{THH}(B)\right)$ and passing to $C_{p^{-}}$-fixed points, we obtain the canonical map $\Gamma$ : $\operatorname{THH}(B)^{C_{p}} \rightarrow \operatorname{THH}(B)^{h C_{p}}$ comparing fixed and homotopy fixed points.

When $B=S$ is the sphere spectrum, $\operatorname{THH}(S)$ and the $\mathbb{T}$-equivariant sphere spectrum are $C_{p^{-}}$ equivariantly equivalent, so the Segal conjecture for $C_{p}$ is the assertion that $\Gamma: \operatorname{THH}(S)^{C_{p}} \rightarrow \operatorname{THH}(S)^{h C_{p}}$ is a $p$-adic equivalence, which was proven in 22 and 2$]$. Our main theorem generalizes this result to the cases when $B=M U$ is the complex cobordism spectrum or $B=B P$ is the $p$-local Brown-Peterson spectrum.

Theorem 1.1. The maps

$$
\Gamma: \operatorname{THH}(M U)^{C_{p}} \longrightarrow \operatorname{THH}(M U)^{h C_{p}}
$$

and

are $p$-adic equivalences.

$$
\Gamma: \operatorname{THH}(B P)^{C_{p}} \longrightarrow \operatorname{THH}(B P)^{h C_{p}}
$$

The corresponding assertion is not true as stated for general symmetric ring spectra $B$, but there are special cases for which it is approximately true. For example, when $B=H \mathbb{F}_{p}, H \mathbb{Z}$ (the EilenbergMac Lane spectra) or $\ell$ (the Adams summand of connective topological $K$-theory $k u$ ) the map $\Gamma$ induces an isomorphism of homotopy groups in sufficiently high degrees, with suitable coefficients, as proved in [19], 11] and 4, respectively. In particular, in those cases the homotopy fixed point spectrum is not connective, even if the fixed point spectrum is. We believe that the explanation for this phenomenon is related to the conjectured "red-shift" property of topological cyclic homology and algebraic $K$-theory $[\underline{5}$. In the examples mentioned above the difference between the fixed and homotopy fixed points stems from the first chromatic truncation present in $B$ (a Milnor $Q^{i}$ that acts trivially on $H^{*}\left(B ; \mathbb{F}_{p}\right)$, followed by a

Date: April 19th 2011. 
$Q^{i+1}$ that acts nontrivially), while $S, M U$ and $B P$ are not truncated at any finite chromatic complexity. The $p$-adic equivalences discussed above are thus rather special properties of the symmetric ring spectra $S, M U$ and BP. This is reflected in our proof of Theorem 1.1, which depends on calculational facts particular to these cases.

Let $C_{p^{n}} \subset \mathbb{T}$ be the cyclic subgroup of order $p^{n}$. The original Segal conjecture for $C_{p^{n}}$ is known to follow from the one for $C_{p}$, without further explicit computations [27, much as the Segal conjecture for general $p$-groups follows from the elementary abelian case [15]. Similarly, combining [12, 1.8] with Theorem 1.1 implies the following.

Corollary 1.2. The maps

and

$$
\Gamma_{n}: \operatorname{THH}(M U)^{C_{p^{n}}} \rightarrow \mathrm{THH}(M U)^{h C_{p^{n}}}
$$

$$
\Gamma_{n}: \operatorname{THH}(B P)^{C_{p^{n}}} \rightarrow \mathrm{THH}(B P)^{h C_{p^{n}}}
$$

are $p$-adic equivalences, for all $n \geq 1$.

1.1. Organization. In Section 2 we outline how to deduce the main theorem from the existence of a suitable bicontinuous isomorphism $\Phi_{B}$, for $B=M U$ or $B P$. This isomorphism is constructed in Section 7 , but this requires computational control of a canonical map $\gamma: \mathrm{THH}(B) \rightarrow \mathrm{THH}(B)^{t C_{p}}$. It suffices to gain this control on the image of a map $\omega: \mathbb{T} \ltimes B \rightarrow \mathrm{THH}(B)$. For this we use a diagram (2.4), which is constructed in Section 5. Thus $\gamma \circ \omega$ factors as the composite of a map $\rho \wedge \epsilon_{B}: \mathbb{T} \ltimes B \rightarrow$ $\mathbb{T} / C_{p} \ltimes R_{+}(B)$, which was analyzed in our paper [23, and a map $\omega^{t}: \mathbb{T} / C_{p} \ltimes R_{+}(B) \rightarrow \mathrm{THH}(B)^{t C_{p}}$, which is constructed and calculated in Sections 3 and 4 . The calculations that are special to $B=M U$ and $B P$ are completed in Section 6.

1.2. History. This work started out as a part of the first author's PhD thesis at the University of Oslo, supervised by the second author. The original thesis covered the case of $B P$ at $p=2$, under the assumption that $B P$ is an $E_{\infty}$ ring spectrum. The current version does not make that assumption, and covers both of the cases $M U$ and $B P$, at all primes.

\section{Strategy of Proof}

In this section we outline how to deduce the Main Theorem 1.1 from the cohomological Theorem 2.1 or equivalently, from the homological Theorem 2.2

Let $\widetilde{E T}$ be the mapping cone of the collapse map $c: E \mathbb{T}_{+} \rightarrow S^{0}$. There is a homotopy cartesian square of $\mathbb{T}$-equivariant spectra

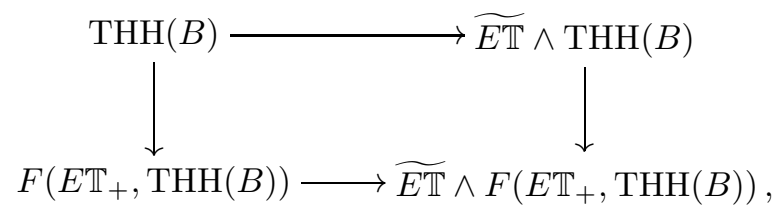

see [18. Passing to $C_{p}$-fixed points, we get a homotopy cartesian square of $\mathbb{T} / C_{p}$-equivariant spectra

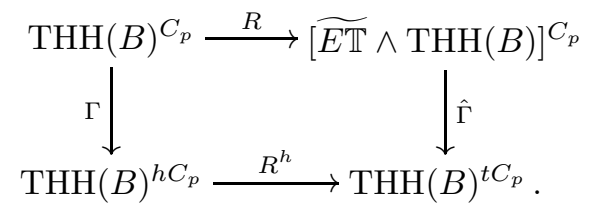

Here $\operatorname{THH}(B)^{t C_{p}}$ denotes the $C_{p}$-Tate construction on $\mathrm{THH}(B)$. In order to prove Theorem 1.1 we use the homotopy cartesian square (2.1) to translate the problem into a question about the map $\hat{\Gamma}:[\widetilde{E T} \wedge$ $\mathrm{THH}(B)]^{C_{p}} \rightarrow \mathrm{THH}(B)^{t C_{p}}$.

Suppose hereafter that $B$ is connective, meaning that $\pi_{*}(B)=0$ for $*<0$. By the cyclotomic structure of $\operatorname{THH}(B)$, see $\left[19\right.$, there is a natural equivalence $[\widetilde{E T} \wedge \operatorname{THH}(B)]^{C_{p}} \stackrel{\simeq}{\longrightarrow} \operatorname{THH}(B)$, and we are faced with the problem of showing that the composite stable map

$$
\gamma: \operatorname{THH}(B) \longrightarrow \operatorname{THH}(B)^{t C_{p}}
$$

is a $p$-adic equivalence for $B=M U$ and $B P$. We do this using the methods of [16] and [23, by realizing the Tate construction $\mathrm{THH}(B)^{t C_{p}}$ as the homotopy limit

$$
\operatorname{THH}(B)^{t C_{p}} \simeq \underset{n}{\operatorname{holim}} \operatorname{THH}(B)^{t C_{p}}[n]
$$


of a Tate tower $\left\{\operatorname{THH}(B)^{t C_{p}}[n]\right\}_{n}$ (see (4.5)) of bounded below spectra of finite type mod $p$, and comparing the Adams spectral sequence

$$
E_{2}^{s, t}=\operatorname{Ext}_{\mathscr{A}}^{s, t}\left(H^{*}(\operatorname{THH}(B)), \mathbb{F}_{p}\right) \Longrightarrow \pi_{t-s} \operatorname{THH}(B)_{p}
$$

to the inverse limit of Adams spectral sequences

$$
E_{2}^{s, t}=\operatorname{Ext}_{\mathscr{A}}^{s, t}\left(H_{c}^{*}\left(\operatorname{THH}(B)^{t C_{p}}\right), \mathbb{F}_{p}\right) \Longrightarrow \pi_{t-s}\left(\operatorname{THH}(B)^{t C_{p}}\right)_{p}
$$

associated to that tower. We always use $\mathbb{F}_{p}$-coefficients for homology and cohomology, $\mathscr{A}$ denotes the Steenrod algebra, and

$$
H_{c}^{*}\left(\operatorname{THH}(B)^{t C_{p}}\right)=\operatorname{colim}_{n} H^{*}\left(\operatorname{THH}(B)^{t C_{p}}[n]\right)
$$

is the continuous cohomology of the Tate tower. We note, as in [23, 2.3], that this colimit depends on the actual tower, not just on its homotopy limit. The key computational input is now that, in the cases $B=S, M U$ and $B P$, the induced $\mathscr{A}$-module homomorphism

$$
\gamma^{*}: H_{c}^{*}\left(\mathrm{THH}(B)^{t C_{p}}\right) \longrightarrow H^{*}(\mathrm{THH}(B))
$$

is an Ext-equivalence, in the sense that it induces an isomorphism between the $E_{2}$-terms of the spectral sequences (2.2) and (2.3). Both spectral sequences are strongly convergent for connective $B$ of finite type $\bmod p$, so this implies that $\gamma, \hat{\Gamma}$ and $\Gamma$ are $p$-adic equivalences. More precisely, we can prove the following theorem. Recall from [2], 23] that the Singer construction on an $\mathscr{A}$-module $M$ is an $\mathscr{A}$-module $R_{+}(M)$, which comes equipped with a natural Ext-equivalence $\epsilon: R_{+}(M) \rightarrow M$.

Theorem 2.1. When $B=S, M U$ or $B P$ there is an $\mathscr{A}$-module isomorphism

$$
\Phi_{B}^{*}: H_{c}^{*}\left(\mathrm{THH}(B)^{t C_{p}}\right) \stackrel{\cong}{\longrightarrow} R_{+}\left(H^{*}(\mathrm{THH}(B))\right)
$$

such that $\gamma^{*}=\epsilon \circ \Phi_{B}^{*}$.

As summarized above, this implies Theorem 1.1. When $B=S$, the 0-simplex inclusion $\eta_{p}: S^{\wedge p} \rightarrow$ $\operatorname{sd}_{p} \operatorname{THH}(S) \cong \operatorname{THH}(S)$ (see Definition 3.6) is a $C_{p}$-equivariant equivalence, so the theorem is already covered by [23, 5.13], or the original proofs of the Segal conjecture. On the other hand, the cases $B=M U$ and $B P$ involve new ideas. For one thing, we will make use of the ring spectrum structure on $\operatorname{THH}(B)^{t C_{p}}$, which means that it is more convenient to work with the continuous homology

$$
H_{*}^{c}\left(\operatorname{THH}(B)^{t C_{p}}\right)=\lim _{n} H_{*}\left(\operatorname{THH}(B)^{t C_{p}}[n]\right)
$$

of the Tate tower, and its induced algebra structure, than with the continuous cohomology. The continuous homology must be viewed as a topological graded $\mathbb{F}_{p}$-vector space, with the linear topology generated by the neighborhood basis of the origin given by the kernels

$$
F_{n} H_{*}^{c}\left(\operatorname{THH}(B)^{t C_{p}}\right)=\operatorname{ker}\left(H_{*}^{c}\left(\operatorname{THH}(B)^{t C_{p}}\right) \rightarrow H_{*}\left(\operatorname{THH}(B)^{t C_{p}}[n]\right)\right) .
$$

We may also view $H_{*}^{c}\left(\mathrm{THH}(B)^{t C_{p}}\right)$ as the limit of its quotients

$$
F^{n} H_{*}^{c}\left(\operatorname{THH}(B)^{t C_{p}}\right)=\operatorname{im}\left(H_{*}^{c}\left(\operatorname{THH}(B)^{t C_{p}}\right) \rightarrow H_{*}\left(\operatorname{THH}(B)^{t C_{p}}[n]\right)\right) .
$$

Let $\mathscr{A}_{*}$ denote the dual of the Steenrod algebra. The $\mathscr{A}_{*}$-comodule structure on $H_{*}\left(\operatorname{THH}(B)^{t C_{p}}[n]\right)$ for each integer $n$ makes the limit $H_{*}^{c}\left(\operatorname{THH}(B)^{t C_{p}}\right)$ a complete $\mathscr{A}_{*}$-comodule in the sense of [23, 2.7]. Similarly, for each $\mathscr{A}_{*}$-comodule $M_{*}$ that is bounded below and of finite type, the homological Singer construction $R_{+}\left(M_{*}\right)$ from [23, 3.7] is a complete $\mathscr{A}_{*}$-comodule, equipped with a natural continuous homomorphism $\epsilon_{*}: M_{*} \rightarrow R_{+}\left(M_{*}\right)$ of complete $\mathscr{A}_{*}$-comodules. The linear topologies on $H_{*}^{c}\left(\mathrm{THH}(B)^{t C_{p}}\right)$ and $R_{+}\left(M_{*}\right)$ allow us to recover the continuous cohomology and the (cohomological) Singer construction, respectively, as their continuous $\mathbb{F}_{p}$-linear duals

$$
\begin{aligned}
H_{c}^{*}\left(\operatorname{THH}(B)^{t C_{p}}\right) & \cong \operatorname{Hom}^{c}\left(H_{*}^{c}\left(\operatorname{THH}(B)^{t C_{p}}\right), \mathbb{F}_{p}\right) \\
R_{+}(M) & \cong \operatorname{Hom}^{c}\left(R_{+}\left(M_{*}\right), \mathbb{F}_{p}\right)
\end{aligned}
$$

where $M=H^{*}(\mathrm{THH}(B))$ and $M_{*}=H_{*}(\mathrm{THH}(B))$, see $\left.23,2.6,2.9\right]$. The corresponding assertions for the full linear duals would be false, since neither $H_{c}^{*}\left(\mathrm{THH}(B)^{t C_{p}}\right)$ nor $R_{+}(M)$ will be of finite type. The $\mathscr{A}$-module homomorphism $\gamma^{*}$ is the continuous dual of the complete $\mathscr{A}_{*}$-comodule homomorphism

$$
\gamma_{*}: H_{*}(\mathrm{THH}(B)) \longrightarrow H_{*}^{c}\left(\mathrm{THH}(B)^{t C_{p}}\right),
$$

where $H_{*}(\mathrm{THH}(B))$ has the discrete topology, and similarly $\epsilon$ is the continuous dual of $\epsilon_{*}$. Theorem 2.1 now follows immediately from its homological analogue, stated below, by letting $\Phi_{B}^{*}$ be the continuous dual of $\Phi_{B}$. 
Theorem 2.2. When $B=S, M U$ or $B P$ there is a continuous isomorphism of complete $\mathscr{A}_{*}$-comodules

$$
\Phi_{B}: R_{+}\left(H_{*}(\mathrm{THH}(B))\right) \stackrel{\cong}{\longrightarrow} H_{*}^{c}\left(\mathrm{THH}(B)^{t C_{p}}\right),
$$

with continuous inverse, such that $\gamma_{*}=\Phi_{B} \circ \epsilon_{*}$.

This is then the main technical result that we will need to prove. The argument relies on computations, and is not of a formal nature. To effect these computations, we establish in Theorem 5.3 that for connective symmetric ring spectra $B$ there is a natural commutative square

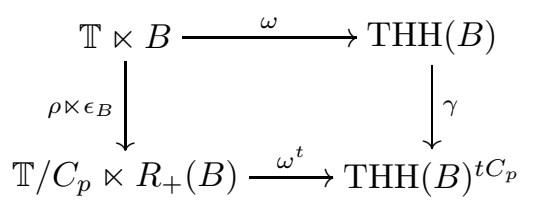

in the stable homotopy category, where $\gamma$ is as above, $\rho: \mathbb{T} \rightarrow \mathbb{T} / C_{p}$ is the $p$-th root isomorphism of groups, $R_{+}(B)=\left(B^{\wedge p}\right)^{t C_{p}}$ is the topological Singer construction from [23, 5.8] realizing the homological Singer construction in continuous homology, and $\epsilon_{B}: B \rightarrow R_{+}(B)$ is a natural map inducing the continuous homomorphism $\epsilon_{*}: H_{*}(B) \rightarrow R_{+}\left(H_{*}(B)\right) \cong H_{*}^{c}\left(R_{+}(B)\right)$ of complete $\mathscr{A}_{*}$-comodules. The map $\omega$ extends the inclusion of 0 -simplices $\eta: B \rightarrow \mathrm{THH}(B)$ using the $\mathbb{T}$-action on the target, and the construction of $\omega^{t}$ is similar, but more elaborate.

To compute the continuous homology of $\operatorname{THH}(B)^{t C_{p}}$, we use the homological Tate spectral sequence

$$
\widehat{E}_{*, *}^{2}=\widehat{H}^{-*}\left(C_{p} ; H_{*}(\mathrm{THH}(B))\right) \Longrightarrow H_{*}^{c}\left(\mathrm{THH}(B)^{t C_{p}}\right)
$$

recalled in Proposition 4.1. In the cases we are interested in, $H_{*}(\mathrm{THH}(B))$ is generated as an algebra by the image of $\omega_{*}$, so we can use the commutative square (2.4) above, known formulas [23, §3.2.1] for $\epsilon_{*}$, and new explicit formulas established in Theorem 4.11 for $\omega_{*}^{t}$, in order to understand the completed $\mathscr{A}_{*}$ comodule homomorphism $\gamma_{*}$. These calculations are carried out for $B=M U$ and $B P$ in Propositions 6.3 and 6.8 and in Theorems 6.4 and 6.9. The proof of Theorem 2.2 is completed in Section 7 the main steps being Propositions 7.2 and 7.3 .

\section{EqUiVARIANT APPROXIMATIONS}

In this section we specify our model for $\operatorname{THH}(B)$ in Definition 3.1, and obtain a natural map

$$
\omega_{p}: \mathbb{T} \ltimes_{C_{p}} B^{\wedge p} \rightarrow \operatorname{sd}_{p} \mathrm{THH}(B)
$$

to its $p$-fold edgewise subdivision in Definition 3.6. In the subsequent lemmas we analyze its effect in homology, and in the Bökstedt spectral sequence $H H_{*}\left(H_{*}(B)\right) \Longrightarrow H_{*}(\mathrm{THH}(B))$. We assume some familiarity with equivariant spectra [21, symmetric ring spectra [20, edgewise subdivision of cyclic objects [10, §1], and the topological Hochschild homology spectrum [19, §2.4].

Recall Connes' extension $\Lambda$ of the category $\Delta$. A cyclic space $X_{\bullet}$ is a contravariant functor from $\Lambda$ to spaces, and its geometric realization $\left|X_{\bullet}\right|$ has a natural $\mathbb{T}$-action. The $\mathbb{T}$-action on a 0 -simplex $x$ in $X_{0}$ traces out the closed loop in $\left|X_{\bullet}\right|$ given by the 1 -simplex $t_{1} s_{0}(x)$ in $X_{1}$.

Let $B$ be a symmetric ring spectrum, with $n$-th space $B_{n}$ for each $n \geq 0$, and let $I$ be Bökstedt's category [9] of finite sets $\mathbf{n}=\{1,2, \ldots, n\}$ for $n \geq 0$ and injective functions.

Definition 3.1. For each $k \geq 0$ and each finite-dimensional $\mathbb{T}$-representation $V$ let

$$
\operatorname{thh}\left(B ; S^{V}\right)_{k}=\underset{\left(\mathbf{n}_{0}, \ldots, \mathbf{n}_{k}\right) \in I^{k+1}}{\operatorname{hocolim}} \operatorname{Map}\left(S^{n_{0}} \wedge \cdots \wedge S^{n_{k}}, B_{n_{0}} \wedge \cdots \wedge B_{n_{k}} \wedge S^{V}\right)
$$

These spaces combine to a cyclic $\operatorname{space} \operatorname{thh}\left(B ; S^{V}\right)_{\bullet}$, with Hochschild-style structure maps $d_{i}, s_{j}$ and $t_{k}$. Its geometric realization $\operatorname{thh}\left(B ; S^{V}\right)=\left|\operatorname{thh}\left(B ; S^{V}\right) \bullet\right|$ has a natural T-action, given as the diagonal of the $\mathbb{T}$-action coming from the cyclic structure and the $\mathbb{T}$-action on $S^{V}$. These $\mathbb{T}$-spaces assemble to a $\mathbb{T}$-equivariant prespectrum $\operatorname{thh}(B)$, with $V$-th space $\operatorname{thh}(B)(V)=\operatorname{thh}\left(B ; S^{V}\right)$. We let

$$
\operatorname{THH}(B)=L\left(\operatorname{thh}(B)^{\tau}\right)
$$

be the genuine $\mathbb{T}$-spectrum obtained by spectrification from a functorial good thickening of this prespectrum. (See [19, §A.1] for the notion of a good prespectrum, and a functorial construction of such a good thickening.) 
Definition 3.2. Let $B_{\text {pre }}^{\wedge 1}$ be the prespectrum with $V$-th space

$$
B_{\text {pre }}^{\wedge 1}(V)=\operatorname{thh}\left(B ; S^{V}\right)_{0}=\underset{\mathbf{n} \in I}{\operatorname{hocolim}} \operatorname{Map}\left(S^{n}, B_{n} \wedge S^{V}\right),
$$

and let $B^{\wedge 1}=L\left(\left(B_{\text {pre }}^{\wedge 1}\right)^{\tau}\right)$. The inclusion of 0 -simplices defines a natural map

$$
\eta: B^{\wedge 1} \rightarrow \mathrm{THH}(B)
$$

of spectra. Using the $\mathbb{T}$-action on the target, we can uniquely extend $\eta$ to a map

$$
\omega: \mathbb{T} \ltimes B^{\wedge 1} \rightarrow \operatorname{THH}(B)
$$

of T-equivariant spectra, as in [21, II.4.1].

We suppose hereafter that $B$ is connective. Without loss of generality we may also assume that $B$ is flat and convergent, as defined in [23, §5.2]. Then the natural maps

$$
B_{\text {pre }}^{\wedge 1}(V) \stackrel{\simeq}{\longleftarrow} B_{\text {pre }}^{\wedge 1}(V)^{\tau} \stackrel{\simeq}{\longrightarrow} B^{\wedge 1}(V)
$$

and

$$
\operatorname{thh}(B)(V) \stackrel{\simeq}{\longleftarrow} \operatorname{thh}(B)(V)^{\tau} \stackrel{\simeq}{\longrightarrow} \operatorname{THH}(B)(V)
$$

are $C$-equivariant equivalences for each finite subgroup $C \subset \mathbb{T}$, by [19, Prop. 2.4]. Furthermore, there is a natural chain of weak equivalences relating $B^{\wedge 1}$ and the Lewis-May spectrum associated to $B$, see 23, 5.5]. Hence we shall simply write $B$ for $B^{\wedge 1}$, especially in the context of the maps $\eta: B \rightarrow \mathrm{THH}(B)$ and $\omega: \mathbb{T} \ltimes B \rightarrow \mathrm{THH}(B)$.

For any simplicial space $X_{\bullet}$, its $p$-fold edgewise subdivision $\operatorname{sd}_{p} X_{\bullet}$ is obtained by precomposing the contravariant functor $X_{\bullet}$ with the functor

$$
\operatorname{sd}_{p}: \Delta \rightarrow \Delta
$$

that takes the object $[k]=\{0<1<\cdots<k\}$ to its $p$-fold concatenation

$$
\operatorname{sd}_{p}([k])=[k] \sqcup \cdots \sqcup[k]=[p(k+1)-1],
$$

for each $k \geq 0$, and likewise for morphisms: $\operatorname{sd}_{p}(f)=f \sqcup \cdots \sqcup f$. Hence $\left(\operatorname{sd}_{p} X\right)_{k}=X_{p(k+1)-1}$. There is a natural homeomorphism [10, 1.1]

$$
D:\left|\operatorname{sd}_{p} X_{\bullet}\right| \stackrel{\cong}{\longrightarrow}\left|X_{\bullet}\right|
$$

induced by the diagonal embeddings $\Delta^{k} \rightarrow \Delta^{p(k+1)-1}$ given in barycentric coordinates as

$$
u \mapsto(u / p, \ldots, u / p)
$$

for $u=\left(u_{0}, \ldots, u_{k}\right) \in \Delta^{k}$, with $\sum_{i} u_{i}=1, u_{i} \geq 0$. In more detail, this is the map

$$
D: \coprod_{k \geq 0}\left(\operatorname{sd}_{p} X\right)_{k} \times \Delta^{k} / \sim \longrightarrow \coprod_{\ell \geq 0} X_{\ell} \times \Delta^{\ell} / \sim
$$

that takes the $k$-th summand to the $\ell$-th one, with $\ell=p(k+1)-1$, via the identity $\left(\operatorname{sd}_{p} X\right)_{k}=X_{\ell}$ and the given embedding $\Delta^{k} \rightarrow \Delta^{\ell}$.

Definition 3.3. There is a natural simplicial map $e_{\bullet}: \operatorname{sd}_{p} X_{\bullet} \rightarrow X_{\bullet}$, induced by the natural transformation id $\rightarrow \operatorname{sd}_{p}$ of functors $\Delta \rightarrow \Delta$, with components $[k] \rightarrow \operatorname{sd}_{p}([k])$ given by inclusion on the $p$-th (= last) copy of $[k]$, for $k \geq 0$. This is the order-preserving function that omits the $(p-1)(k+1)$ first elements in the target, so in simplicial degree $k$,

$$
e_{k}=d_{0}^{(p-1)(k+1)}:\left(\operatorname{sd}_{p} X\right)_{k}=X_{p(k+1)-1} \rightarrow X_{k}
$$

is equal to the $(p-1)(k+1)$-fold iterate of the 0 -th face map. After geometric realization,

$$
e=\left|e_{\bullet}\right|:\left|\operatorname{sd}_{p} X_{\bullet}\right| \rightarrow\left|X_{\bullet}\right|
$$

is induced by the corresponding iterated face maps $\delta_{0}^{(p-1)(k+1)}: \Delta^{k} \rightarrow \Delta^{p(k+1)-1}$, given in barycentric coordinates as

for $u \in \Delta^{k}$, as above.

$$
u \mapsto(0, \ldots, 0, u)
$$

Lemma 3.4. There is a natural homotopy $D \simeq e=\left|e_{\bullet}\right|$ of $\operatorname{maps}\left|\operatorname{sd}_{p} X_{\bullet}\right| \rightarrow\left|X_{\bullet}\right|$.

Proof. The homotopy is given by the convex linear combination

$$
(t, u) \mapsto(1-t)(u / p, \ldots, u / p)+t(0, \ldots, 0, u)
$$

for $t \in[0,1], u \in \Delta^{k}$, of the two maps $\Delta^{k} \rightarrow \Delta^{p(k+1)-1}$. 
Recall the " $p$-fold cover" $\Lambda_{p} \rightarrow \Lambda$ [10, 1.5]. Roughly, $\Lambda_{p}$ is like Connes' category $\Lambda$, except that the cyclic morphism $\tau_{k}:[k] \rightarrow[k]$ satisfies $\tau_{k}^{p(k+1)}=\mathrm{id}$ instead of $\tau_{k}^{k+1}=\mathrm{id}$. A $\Lambda_{p}$-space is a contravariant functor from $\Lambda_{p}$ to spaces. When $X_{\bullet}$ is a cyclic space, with cyclic operators $t_{k}: X_{k} \rightarrow X_{k}$ satisfying $t_{k}^{k+1}=\mathrm{id}$ for $k \geq 0$, the $p$-fold edgewise subdivision $\operatorname{sd}_{p} X$ • is a $\Lambda_{p}$-space, with operators $t_{k}:\left(\operatorname{sd}_{p} X\right)_{k} \rightarrow$ $\left(\operatorname{sd}_{p} X\right)_{k}$ satisfying $t_{k}^{p(k+1)}=\mathrm{id}$, defined to be equal to the operators $t_{p(k+1)-1}: X_{p(k+1)-1} \rightarrow X_{p(k+1)-1}$.

Lemma 3.5. Let $X_{\bullet}$ be a cyclic space. There is a natural $\mathbb{T}$-action on $\left|\operatorname{sd}_{p} X_{\bullet}\right|$ such that $D:\left|\operatorname{sd}_{p} X_{\bullet}\right| \rightarrow$ $\left|X_{\bullet}\right|$ is a $\mathbb{T}$-equivariant homeomorphism. The action of the subgroup $C_{p} \subset \mathbb{T}$ is simplicial, in the sense that it comes from a $C_{p}$-action on $\operatorname{sd}_{p} X_{\bullet}$, such that a generator of $C_{p}$ acts by

$$
t_{k}^{k+1}:\left(\operatorname{sd}_{p} X\right)_{k} \rightarrow\left(\operatorname{sd}_{p} X\right)_{k}
$$

for $k \geq 0$. In particular, the inclusion of 0 -simplices $\left(\operatorname{sd}_{p} X\right)_{0} \subset\left|\operatorname{sd}_{p} X_{\bullet}\right|$ is $C_{p}$-equivariant.

The $\mathbb{T}$-action on a 0 -simplex $y$ in $\left(\operatorname{sd}_{p} X\right)_{0}=X_{p-1}$ traces out the closed loop in $\left|\operatorname{sd}_{p} X.\right|$ given by the chain of $p$ 1-simplices

$$
t_{1} s_{0}(y), t_{1}^{3} s_{0}(y), \ldots, t_{1}^{2 p-1} s_{0}(y)
$$

in $\left(\operatorname{sd}_{p} X\right)_{1}=X_{2 p-1}$. These meet at the $p$ 0-simplices

$$
y, t_{1}^{2}(y), \ldots, t_{1}^{2 p-2}(y)
$$

that constitute the $C_{p}$-orbit of $y$.

Proof. See [10, 1.6] and the surrounding discussion.

Definition 3.6. Let $\operatorname{sd}_{p} \operatorname{thh}(B)=\left|\operatorname{sd}_{p} \operatorname{thh}(B) \bullet\right|$ be the $\mathbb{T}$-equivariant prespectrum with $V$-th space $\operatorname{sd}_{p} \operatorname{thh}(B)(V)=\left|\operatorname{sd}_{p} \operatorname{thh}\left(B ; S^{V}\right) \bullet\right|$, and let $\operatorname{sd}_{p} \operatorname{THH}(B)=L\left(\operatorname{sd}_{p} \operatorname{thh}(B)^{\tau}\right)$ be the spectrification of its good thickening, as in Definition 3.1 In simplicial degree 0 ,

$$
\operatorname{sd}_{p} \operatorname{thh}\left(B ; S^{V}\right)_{0}=\operatorname{thh}\left(B ; S^{V}\right)_{p-1}=B_{\text {pre }}^{\wedge p}(V)
$$

in the notation of $[23,5.3]$. Hence the inclusion of 0 -simplices defines a natural map

$$
\eta_{p}: B^{\wedge p} \rightarrow \operatorname{sd}_{p} \operatorname{THH}(B)
$$

of $C_{p}$-equivariant spectra, where $B^{\wedge p}=L\left(\left(B_{\text {pre }}^{\wedge p}\right)^{\tau}\right)$. Using the full $\mathbb{T}$-action on the target, we can uniquely extend $\eta_{p}$ to a map

$$
\omega_{p}: \mathbb{T} \ltimes_{C_{p}} B^{\wedge p} \rightarrow \operatorname{sd}_{p} \operatorname{THH}(B)
$$

of $\mathbb{T}$-equivariant spectra, again as in [21, II.4.1].

Combining these constructions, we have a $\mathbb{T}$-equivariant homeomorphism

$$
D: \operatorname{sd}_{p} \operatorname{THH}(B) \stackrel{\cong}{\longrightarrow} \mathrm{THH}(B) \text {. }
$$

Lemma 3.7. The composite

$$
D \circ \eta_{p}: B^{\wedge p} \rightarrow \mathrm{THH}(B)
$$

is homotopic to the composite

$$
e \circ \eta_{p}=\eta \circ d_{0}^{p-1}: B^{\wedge p} \rightarrow \mathrm{THH}(B)
$$

where $d_{0}^{p-1}: B^{\wedge p} \rightarrow B^{\wedge 1} \simeq B$ is the p-fold multiplication map.

Proof. The homotopy is that of Lemma 3.4. The identity $e \circ \eta_{p}=\eta \circ d_{0}^{p-1}$ amounts to the formula (3.1) for $e_{k}$, in the special case $k=0$.

To analyze the extension $\omega_{p}$ of $\eta_{p}$, we pass to homology.

Definition 3.8. The circle $\mathbb{T}$, with the subgroup action of $C_{p}$, is a free $C_{p}$-CW complex. Its $\bmod p$ cellular complex $C_{*}(\mathbb{T})=\mathbb{F}_{p}\left[C_{p}\right]\left\{e_{0}, e_{1}\right\}$ has boundary operator $d\left(e_{1}\right)=(T-1) e_{0}$, where $T \in C_{p}$ is the generator mapping to $\exp (2 \pi i / p) \in \mathbb{T}, e_{1}$ is the 1-cell covering the arc from 1 to $T$, and $e_{0}$ is the 0 -cell 1 .

Let $C_{*}(B)$ be the cellular complex of a $\mathrm{CW}$-approximation to $B$, and choose a quasi-isomorphism $H_{*}(B) \simeq C_{*}(B)$, taking each homology class to a representing cycle in its class. 
Lemma 3.9. There are quasi-isomorphisms

$$
C_{*}\left(\mathbb{T} \ltimes_{C_{p}} B^{\wedge p}\right) \simeq C_{*}(\mathbb{T}) \otimes_{C_{p}} C_{*}(B)^{\otimes p} \simeq C_{*}(\mathbb{T}) \otimes_{C_{p}} H_{*}(B)^{\otimes p}
$$

and natural isomorphisms

$$
\begin{aligned}
H_{*}\left(\mathbb{T} \ltimes_{C_{p}} B^{\wedge p}\right) & \cong H_{*}\left(C_{*}(\mathbb{T}) \otimes_{C_{p}} H_{*}(B)^{\otimes p}\right) \\
& \cong \operatorname{cok}(T-1)\left\{e_{0}\right\} \oplus \operatorname{ker}(T-1)\left\{e_{1}\right\}
\end{aligned}
$$

where

$$
T-1: H_{*}(B)^{\otimes p} \rightarrow H_{*}(B)^{\otimes p}
$$

is the difference between the cyclic permutation, induced by the action of $T \in C_{p}$ on $B^{\wedge p}$, and the identity.

Proof. The first quasi-isomorphism follows from [21, VIII.1.6] and [21, VIII.2.9], combined with cellular approximation. We use [23, 5.5] to compare $B^{\wedge p}$ with the $p$-th external power $B^{(p)}$. The second quasiisomorphism is then clear, since $C_{*}(\mathbb{T})$ is $C_{p}$-free. This implies the first isomorphism. The second isomorphism is simply the computation of the homology of the complex

$$
d: H_{*}(B)^{\otimes p}\left\{e_{1}\right\} \rightarrow H_{*}(B)^{\otimes p}\left\{e_{0}\right\},
$$

where $d$ maps $e_{1} \otimes\left(\alpha_{1} \otimes \cdots \otimes \alpha_{p}\right)$ to $e_{0} \otimes(T-1)\left(\alpha_{1} \otimes \cdots \otimes \alpha_{p}\right)$.

Definition 3.10. For $\alpha \in H_{q}(B)$ let $e_{1} \otimes \alpha^{\otimes p} \in H_{1+p q}\left(\mathbb{T} \ltimes_{C_{p}} B^{\wedge p}\right)$ denote the homology class of $e_{1} \otimes \alpha^{\otimes p}$ in $C_{*}(\mathbb{T}) \otimes_{C_{p}} H_{*}(B)^{\otimes p}$, and let

$$
\alpha^{p-1} \wedge \alpha=\left(D \circ \omega_{p}\right)_{*}\left(e_{1} \otimes \alpha^{\otimes p}\right) \in H_{1+p q}(\operatorname{THH}(B))
$$

denote its image under $D \circ \omega_{p}: \mathbb{T} \ltimes_{C_{p}} B^{\wedge p} \rightarrow \operatorname{THH}(B)$.

The following spectral sequence was used by Bökstedt $[9$ to compute $\operatorname{THH}(\mathbb{Z} / p)$ and $\operatorname{THH}(\mathbb{Z})$. See [25, 3.1], [3, §4] for more background.

Lemma 3.11. In the Bökstedt spectral sequence

$$
E_{*, *}^{2}=H H_{*}\left(H_{*}(B)\right) \Longrightarrow H_{*}(\mathrm{THH}(B))
$$

the class $\alpha^{p-1} \wedge \alpha$ is represented modulo filtration 0 by the homology class of the Hochschild 1-cycle

$$
\alpha^{p-1} \otimes \alpha \in H_{*}(B) \otimes H_{*}(B) .
$$

Proof. By Lemma 3.4 we may compute $\alpha^{p-1} \wedge \alpha$ as the image of $e_{1} \otimes \alpha^{\otimes p}$ under the cellular map $e \circ \omega_{p}$. By the second part of Lemma 3.5 and the formula (3.1) for $e=\left|e_{\bullet}\right|$ in simplicial degree $k=1$, the cycle $e_{1} \otimes \alpha^{\otimes p}$ maps under $e \circ \omega_{p}$ to the cycle

$$
d_{0}^{2 p-2} t_{1} s_{0}\left(\alpha^{\otimes p}\right)=d_{0}^{2 p-2}(1 \otimes \alpha \otimes \cdots \otimes 1 \otimes \alpha)=\alpha^{p-1} \otimes \alpha
$$

in the Bökstedt spectral sequence $E^{1}$-term.

We can be more specific about $\alpha^{p-1} \wedge \alpha$ under additional commutativity hypotheses. The homotopy cofiber sequence

$$
B \stackrel{i}{\longrightarrow} \mathbb{T} \ltimes B \stackrel{j}{\longrightarrow} S^{1} \wedge B
$$

is stably split by the collapse map $c: \mathbb{T} \ltimes B \rightarrow B$, and its homotopy fiber map, a section $s: S^{1} \wedge B \rightarrow \mathbb{T} \ltimes B$. We let $\sigma: S^{1} \wedge B \rightarrow \operatorname{THH}(B)$ be the composite map

$$
S^{1} \wedge B \stackrel{s}{\longrightarrow} \mathbb{T} \ltimes B \stackrel{\omega}{\longrightarrow} \mathrm{THH}(B) .
$$

For $\alpha \in H_{q}(B)$, the homology class $\sigma \alpha=\sigma_{*}(\alpha) \in H_{1+q}(\mathrm{THH}(B))$ is represented by the Hochschild 1-cycle $1 \otimes \alpha \in H_{*}(B) \otimes H_{*}(B)$ in the Bökstedt spectral sequence above, see [25, 3.2].

When $B$ is an $E_{2}$ symmetric ring spectrum, it follows from [13] that $\operatorname{THH}(B)$ is an $E_{1}=A_{\infty}$ ring spectrum, hence equivalent to an (associative) $S$-algebra. Furthermore, the Bökstedt spectral sequence is an algebra spectral sequence, with product induced by the shuffle product on the Hochschild complex. See e.g. [3, 4.2].

Lemma 3.12. Suppose that $B$ is an $E_{2}$ symmetric ring spectrum. Then for $\alpha \in H_{q}(B)$ we have

$$
\alpha^{p-1} \wedge \alpha \equiv \alpha^{p-1} \cdot \sigma \alpha
$$

in $H_{*}(\operatorname{THH}(B))$, modulo the image of $\eta_{*}: H_{*}(B) \rightarrow H_{*}(\operatorname{THH}(B))$. 
Proof. The shuffle product of the 0 -cycle $\alpha^{p-1}$ and the 1 -cycle $1 \otimes \alpha$ is the 1 -cycle $\alpha^{p-1} \otimes \alpha$. Hence the product $\alpha^{p-1} \cdot \sigma \alpha$ and $\alpha^{p-1} \wedge \alpha$ have the same representative in Hochschild filtration 1 , and must be equal modulo the image of Hochschild filtration 0.

The following corollary applies to $B=S, M U$ and $B P$. (According to Basterra and Mandell [7, 8], $B P$ is an $E_{4}$ ring spectrum.)

Corollary 3.13. Suppose that $B$ is an $E_{2}$ symmetric ring spectrum with $H_{*}(B)$ concentrated in even degrees. Then for $\alpha \in H_{q}(B)$ we have

$$
\alpha^{p-1} \wedge \alpha=\alpha^{p-1} \cdot \sigma \alpha
$$

Proof. There is only something to prove when $q$ is even, in which case $H_{1+p q}(B)=0$ by hypothesis, so the indeterminacy is 0 .

When $B$ is a commutative symmetric ring spectrum, it follows similarly that $\operatorname{THH}(B)$ is an $E_{\infty}$ ring spectrum, hence equivalent to a commutative $S$-algebra. The multiplication maps $\operatorname{THH}(B)_{k} \simeq$ $B^{\wedge(k+1)} \rightarrow B$ then combine to an augmentation map $\epsilon: \operatorname{THH}(B) \rightarrow B$ (not to be confused with the stable map $\epsilon_{B}$ ), such that $\epsilon \eta=\operatorname{id}_{B}$ and $\epsilon \sigma \simeq *$. See e.g. [3, §3]. We can thus use $\epsilon$ to determine the error term in Lemma 3.12 .

The commutative product on $B$ makes the associated Lewis-May spectrum an $E_{\infty}$ ring spectrum, with structure maps

$$
\xi_{p}: E \Sigma_{p} \ltimes_{\Sigma_{p}} B^{(p)} \longrightarrow B
$$

among others. Here $E \Sigma_{p}$ is a free, contractible $\Sigma_{p}$-space. Up to homotopy, there is a unique map $\chi: \mathbb{T} \rightarrow E \Sigma_{p}$ that is equivariant with respect to the inclusion $C_{p} \subset \Sigma_{p}$. Furthermore, the composite map

$$
\mathbb{T} \ltimes_{C_{p}} B^{(p)} \stackrel{\chi \ltimes \mathrm{id}}{\longrightarrow} E \Sigma_{p} \ltimes_{\Sigma_{p}} B^{(p)} \stackrel{\xi_{p}}{\longrightarrow} B
$$

exhibits the commuting homotopy $\mu^{p} \simeq \mu^{p} T$ that is preferred by the $E_{\infty}$ structure, where $\mu^{p}: B^{(p)} \rightarrow B$ is the multiplication map and $T: B^{(p)} \rightarrow B^{(p)}$ is the cyclic permutation. Using [23, 5.5], we can identify the source here with the source $\mathbb{T} \ltimes_{C_{p}} B^{\wedge p}$ of $\omega_{p}$.

Lemma 3.14. Let $B$ be a commutative symmetric ring spectrum. The composite map

$$
\mathbb{T} \ltimes_{C_{p}} B^{\wedge p} \stackrel{\omega_{p}}{\longrightarrow} \operatorname{sd}_{p} \operatorname{THH}(B) \stackrel{D}{\longrightarrow} \operatorname{THH}(B) \stackrel{\epsilon}{\longrightarrow} B
$$

is homotopic to the composite map

$$
\mathbb{T} \ltimes_{C_{p}} B^{\wedge p} \simeq \mathbb{T} \ltimes_{C_{p}} B^{(p)} \stackrel{\chi \ltimes \mathrm{id}}{\longrightarrow} E \Sigma_{p} \ltimes_{\Sigma_{p}} B^{(p)} \stackrel{\xi_{p}}{\longrightarrow} B .
$$

Proof. Using Lemma 3.4 we may replace $D$ by the simplicial map $e=\left|e_{\bullet}\right|$. When restricted to $1 \in C_{p} \subset$ $\mathbb{T}$, both maps then agree with the $p$-fold multiplication map $d_{0}^{p-1}: B^{\wedge p} \rightarrow B$, taking $\alpha_{1} \otimes \cdots \otimes \alpha_{p}$ to $\alpha_{1} \cdots \alpha_{p}$ (in homology). Similarly, when restricted to $T \in C_{p} \subset \mathbb{T}$, both maps agree with the cyclically permuted multiplication map $d_{0}^{p-1} t_{p-1}: B^{\wedge p} \rightarrow B$, taking $\alpha_{1} \otimes \cdots \otimes \alpha_{p}$ to $\alpha_{p} \alpha_{1} \cdots \alpha_{p-1}$. When restricted to the 1-cell $e_{1} \subset \mathbb{T}$, connecting 1 to $T$, both maps exhibit the commuting homotopy between these two maps that is preferred by the given $E_{\infty}$ structure. (This is how the $E_{\infty}$ structure enters into the definition of $\epsilon$.) In particular the homotopies agree, up to homotopy relative to the endpoints.

The following corollary applies to $B=H \mathbb{F}_{p}, H \mathbb{Z}, k u$ and $\ell$.

Corollary 3.15. Let $B$ be a commutative symmetric ring spectrum. For $\alpha \in H_{q}(B)$ we have

$$
\alpha^{p-1} \wedge \alpha=\alpha^{p-1} \cdot \sigma \alpha+\eta_{*}\left(Q_{1}(\alpha)\right)
$$

in $H_{*}(\mathrm{THH}(B))$, where $Q_{1}(\alpha)=\xi_{p *}\left(e_{1} \otimes \alpha^{\otimes p}\right)$ is the image of $\alpha$ under the Dyer-Lashof operation $Q_{1}: H_{q}(B) \rightarrow H_{1+p q}(B)$.

Proof. We know that $\alpha^{p-1} \wedge \alpha=\left(D \omega_{p}\right)_{*}\left(e_{1} \otimes \alpha^{\otimes p}\right)$ equals $\alpha^{p-1} \cdot \sigma \alpha+\eta_{*}(x)$ for some $x \in H_{*}(B)$. Applying $\epsilon_{*}$, we get that $\epsilon_{*}\left(\alpha^{p-1} \wedge \alpha\right)=\left(\epsilon D \omega_{p}\right)_{*}\left(e_{1} \otimes \alpha^{\otimes p}\right)=\xi_{p *}\left(e_{1} \otimes \alpha^{\otimes p}\right)=Q_{1}(\alpha)$ equals $\epsilon_{*}\left(\alpha^{p-1} \cdot \sigma \alpha+\eta_{*}(x)\right)=$ $\alpha^{p-1} \cdot 0+x=x$. 


\section{TATE REPRESENTATIVES}

To prove Theorem 2.2, we use the homological Tate spectral sequence in Proposition 4.1 to study the continuous homology of Tate constructions. We only need to consider the $C_{p}$-equivariant case. Using Proposition 4.9 we deduce formulas in Theorem 4.11 for the effect of a natural map

$$
\omega^{t}: \mathbb{T} / C_{p} \ltimes\left(B^{\wedge p}\right)^{t C_{p}} \rightarrow \mathrm{THH}(B)^{t C_{p}},
$$

as seen by the eyes of the Tate spectral sequence. These formulas will be essential for the calculations in Section 6.

Proposition 4.1. Let $X$ be a $C_{p}$-equivariant spectrum. Assume that $X$ is bounded below with $H_{*}(X)$ of finite type. Then the homological Tate spectral sequence

$$
\widehat{E}_{s, t}^{2}(X)=\widehat{H}^{-s}\left(C_{p} ; H_{t}(X)\right) \Longrightarrow H_{s+t}^{c}\left(X^{t C_{p}}\right)
$$

converges strongly to the continuous homology of $X^{t C_{p}}$ as a complete $\mathscr{A}_{*}$-comodule.

If, furthermore, $X$ is a $C_{p}$-equivariant ring spectrum, then this is an algebra spectral sequence, whose product at the $\widehat{E}^{2}$-term is given by the cup product in Tate cohomology and the Pontryagin product on $H_{*}(X)$.

Proof. See Propositions 4.15 and 4.17 in 23 .

Remark 4.2. The strong convergence of the spectral sequence refers to the complete Hausdorff filtration of the abutment $H_{*}^{c}\left(X^{t C_{p}}\right)$ by the kernels of the natural homomorphisms induced by the maps $X^{t C_{p}} \rightarrow$ $X^{t C_{p}}[n]$, see (4.5) below. We shall refer to this increasing filtration on $H_{*}^{c}\left(X^{t C_{p}}\right)$ as the Tate filtration.

When $B$ is a connective symmetric ring spectrum, with $H_{*}(B)$ of finite type, then both $X=B^{\wedge p}$ and $X=\operatorname{THH}(B)$ are $C_{p}$-spectra that are bounded below with $H_{*}(X)$ of finite type. This is clear from the Künneth formula and the Bökstedt spectral sequence in Lemma 3.11

Remark 4.3. In the case when $X=S^{\wedge p} \cong \operatorname{THH}(S)$ is the $C_{p}$-equivariant sphere spectrum, $H_{*}(X)=\mathbb{F}_{p}$ is concentrated in degree 0 , so

$$
\widehat{E}_{*, *}^{2}\left(S^{\wedge p}\right)=\widehat{H}^{-*}\left(C_{p} ; \mathbb{F}_{p}\right)
$$

is concentrated on the horizontal axis. Here $\widehat{H}^{-*}\left(C_{p} ; \mathbb{F}_{p}\right)=P\left(u, u^{-1}\right)$ for $p=2$ and $\widehat{H}^{-*}\left(C_{p} ; \mathbb{F}_{p}\right)=$ $E(u) \otimes P\left(t, t^{-1}\right)$ for $p$ odd, with $u \in \widehat{H}^{1}$ and $t \in \widehat{H}^{2}$. There cannot be any differentials in this spectral sequence, for bidegree reasons, so each class $u^{i} t^{r}$ is an infinite cycle. (This formula applies when $p$ is odd - the reader should always replace $u^{i} t^{r}$ by $u^{i+2 r}$ when $p=2$.)

By naturality with respect to the unit maps $S^{\wedge p} \rightarrow B^{\wedge p}$ and $\operatorname{THH}(S) \rightarrow \operatorname{THH}(B)$ it follows that the classes $u^{i} t^{r}$ are infinite cycles, also in the homological Tate spectral sequences for $X=B^{\wedge p}$ and $X=\operatorname{THH}(B)$. Hence all of these spectral sequences exhibit a horizontal periodicity, with each $\widehat{E}^{r}$-term being determined by the part on the vertical axis via an isomorphism

$$
\widehat{E}_{*, *}^{r}(X) \cong \widehat{H}^{-*}\left(C_{p} ; \mathbb{F}_{p}\right) \otimes \widehat{E}_{0, *}^{r}(X) .
$$

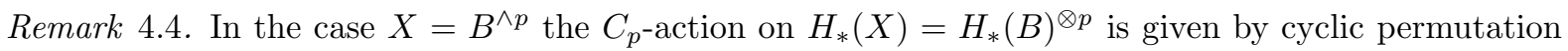
of the tensor factors, so

$$
\begin{aligned}
\widehat{E}_{*, *}^{2}\left(B^{\wedge p}\right) & =\widehat{H}^{-*}\left(C_{p} ; H_{*}(B)^{\otimes p}\right) \cong \widehat{H}^{-*}\left(C_{p} ; \mathbb{F}_{p}\right) \otimes \mathbb{F}_{p}\left\{\alpha^{\otimes p}\right\} \\
& \Longrightarrow H_{*}^{c}\left(\left(B^{\wedge p}\right)^{t C_{p}}\right),
\end{aligned}
$$

where $\alpha$ ranges over an $\mathbb{F}_{p}$-basis of $H_{*}(B)$. Also in this case the spectral sequence collapses at the $\widehat{E}^{2}$-term, and converges to

$$
H_{*}^{c}\left(\left(B^{\wedge p}\right)^{t C_{p}}\right)=H_{*}^{c}\left(R_{+}(B)\right) \cong R_{+}\left(H_{*}(B)\right),
$$

see [23, 5.14]. Here $R_{+}(B)=\left(B^{\wedge p}\right)^{t C_{p}}$ is the topological Singer construction on $B$, and $R_{+}\left(H_{*}(B)\right)$ is the homological Singer construction on $H_{*}(B)$, discussed in Definitions 5.8 and 3.7 of [23], respectively.

The right hand isomorphism is given in Theorem 5.9 of that paper. Implicit in this isomorphism is the fact that for each $u^{i} t^{r} \in \widehat{H}^{-*}\left(C_{p} ; \mathbb{F}_{p}\right)$ and $\alpha \in H_{*}(B)$ there is a preferred class in $H_{*}^{c}\left(\left(B^{\wedge p}\right)^{t C_{p}}\right)$ that is represented in the Tate spectral sequence by $u^{i} t^{r} \otimes \alpha^{\otimes p}$ in $\widehat{E}_{*, *}^{2}\left(B^{\wedge p}\right)$. It is obtained by representing $\alpha$ by a map $f: S^{q} \rightarrow H \wedge B$, and taking $u^{i} t^{r} \otimes \alpha^{\otimes p}$ to be in the image of an induced homomorphism

$$
H_{*}^{c}\left(\left(f^{p}\right)^{t C_{p}}\right): H_{*}^{c}\left(\left(S^{p q}\right)^{t C_{p}}\right) \rightarrow H_{*}^{c}\left(\left(B^{\wedge p}\right)^{t C_{p}}\right) .
$$

See the proof of [23, 5.14] for details. Hence $u^{i} t^{r} \otimes \alpha^{\otimes p}$ is a well-defined class in $H_{*}^{c}\left(\left(B^{\wedge p}\right)^{t C_{p}}\right)$, not just defined modulo the Tate filtration. 
Remark 4.5. In the case $X=\operatorname{THH}(B)$ the $C_{p}$-action on $H_{*}(X)=H_{*}(\operatorname{THH}(B))$ is obtained by restriction from a $\mathbb{T}$-action, hence is algebraically trivial. Thus

$$
\begin{aligned}
\widehat{E}_{*, *}^{2}(\mathrm{THH}(B)) & =\widehat{H}^{-*}\left(C_{p} ; H_{*}(\mathrm{THH}(B))\right) \cong \widehat{H}^{-*}\left(C_{p} ; \mathbb{F}_{p}\right) \otimes H_{*}(\operatorname{THH}(B)) \\
& \Longrightarrow H_{*}^{c}\left(\operatorname{THH}(B)^{t C_{p}}\right) .
\end{aligned}
$$

In this case the spectral sequence does not generally collapse at the $\widehat{E}^{2}$-term. For example, the $d^{2}$ differential satisfies

$$
d^{2}\left(u^{i} t^{r} \otimes \alpha\right)=u^{i} t^{r+1} \otimes \sigma \alpha
$$

for $p$ odd, and similarly for $p=2$, see [29, 3.3]. When $B$ is an $E_{\infty} \operatorname{ring}$ spectrum, so that $\operatorname{THH}(B)$ is equivalent to a $\mathbb{T}$-equivariant commutative $S$-algebra, there are many cases where this spectral sequence collapses at the $\widehat{E}^{3}$-term, see [14, 1.2]. For example, this is the case for $B=M U$, as we shall prove directly in Proposition 6.3 below.

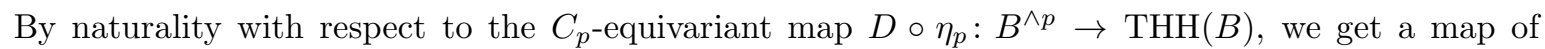
homological Tate spectral sequences

$$
\widehat{E}_{*, *}^{2}\left(B^{\wedge p}\right) \longrightarrow \widehat{E}_{*, *}^{2}(\mathrm{THH}(B))
$$

taking $u^{i} t^{r} \otimes \alpha^{\otimes p}$ to $u^{i} t^{r} \otimes \alpha^{p}$ (recall Lemma 3.7), which converges to a complete $\mathscr{A}_{*}$-comodule homomorphism

$$
\eta_{*}^{t}: H_{*}^{c}\left(\left(B^{\wedge p}\right)^{t C_{p}}\right) \longrightarrow H_{*}^{c}\left(\mathrm{THH}(B)^{t C_{p}}\right) .
$$

This is useful for determining differentials and $\mathscr{A}_{*}$-comodule structure in the target, but there is an even more useful extension of this homomorphism,

$$
\omega_{*}^{t}: H_{*}\left(\mathbb{T} / C_{p}\right) \otimes H_{*}^{c}\left(\left(B^{\wedge p}\right)^{t C_{p}}\right) \longrightarrow H_{*}^{c}\left(\mathrm{THH}(B)^{t C_{p}}\right),
$$

which is constructed by taking the full $\mathbb{T}$-action on $\operatorname{THH}(B)$ into account. We now explain this construction.

Consider the diagram

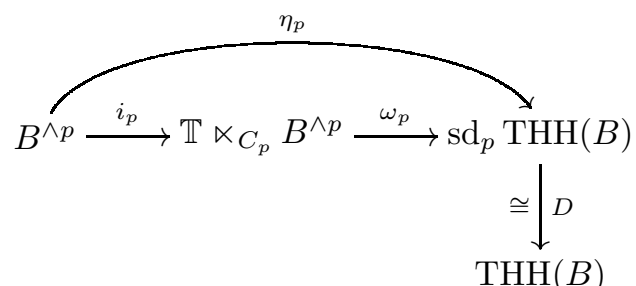

of $C_{p}$-equivariant spectra, where $i_{p}$ is induced by the inclusion $C_{p} \subset \mathbb{T}$. Note that $\omega_{p}$ and $D$ are $\mathbb{T}$ equivariant maps. For any closed subgroup $G \subseteq \mathbb{T}$ containing $C_{p}$, and any $G$-spectrum $X$, the $C_{p}$-Tate construction

$$
X^{t C_{p}}=\left[\widetilde{E T} \wedge F\left(E \mathbb{T}_{+}, X\right)\right]^{C_{p}}
$$

is naturally a $G / C_{p}$-spectrum. Applying the $C_{p}$-Tate construction to (4.1) we can form a commutative diagram:

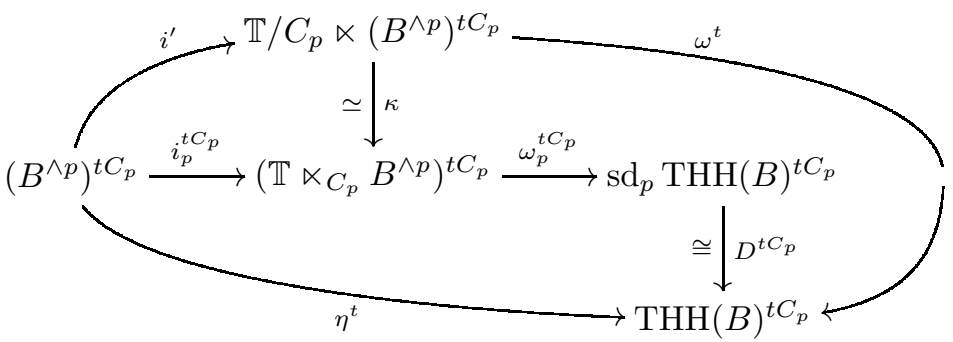

Definition 4.6. Let

be the map $\eta^{t}=\left(D \circ \eta_{p}\right)^{t C_{p}}$, let

$$
\eta^{t}:\left(B^{\wedge p}\right)^{t C_{p}} \rightarrow \mathrm{THH}(B)^{t C_{p}}
$$

$$
\kappa: \mathbb{T} / C_{p} \ltimes\left(B^{\wedge p}\right)^{t C_{p}} \rightarrow\left(\mathbb{T} \ltimes C_{p} B^{\wedge p}\right)^{t C_{p}}
$$

be the unique $\mathbb{T} / C_{p}$-equivariant map that extends $i_{p}^{t C_{p}}$, and let

$$
\omega^{t}: \mathbb{T} / C_{p} \ltimes\left(B^{\wedge p}\right)^{t C_{p}} \rightarrow \operatorname{THH}(B)^{t C_{p}}
$$


be the $\mathbb{T} / C_{p^{-}}$-equivariant composite $\omega^{t}=\left(D \circ \omega_{p}\right)^{t C_{p}} \circ \kappa$.

Lemma 4.7. The map

$$
\kappa: \mathbb{T} / C_{p} \ltimes\left(B^{\wedge p}\right)^{t C_{p}} \stackrel{\simeq}{\longrightarrow}\left(\mathbb{T} \ltimes_{C_{p}} B^{\wedge p}\right)^{t C_{p}}
$$

is an equivalence.

Proof. The $C_{p}$-equivariant cofiber sequence

$$
C_{p+} \stackrel{i_{p}}{\longrightarrow} \mathbb{T}_{+} \stackrel{j_{p}}{\longrightarrow} S^{1} \wedge C_{p+}
$$

induces a map of cofiber sequences

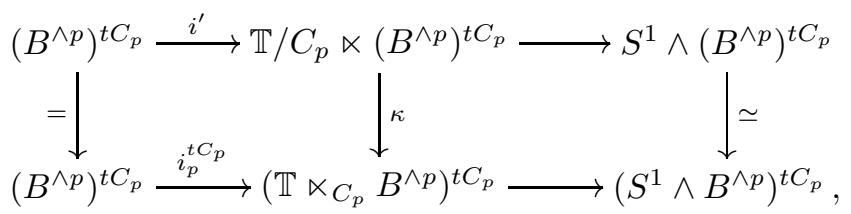

which implies the result.

Remark 4.8. In the intermediate case of the homological Tate spectral sequence for $X=\mathbb{T} \ltimes C_{p} B^{\wedge p}$, the homology $H_{*}(X)=H_{*}\left(\mathbb{T} \ltimes C_{p} B^{\wedge p}\right)$ was given in Lemma 3.9. The $C_{p}$-action extends to a $\mathbb{T}$-action, hence is algebraically trivial. Thus in this case

$$
\begin{aligned}
\widehat{E}_{*, *}^{2}\left(\mathbb{T} \ltimes_{C_{p}} B^{\wedge p}\right) & =\widehat{H}^{-*}\left(C_{p} ; H_{*}\left(\mathbb{T} \ltimes_{C_{p}} B^{\wedge p}\right)\right) \cong \widehat{H}^{-*}\left(C_{p} ; \mathbb{F}_{p}\right) \otimes H_{*}\left(\mathbb{T} \ltimes_{C_{p}} B^{\wedge p}\right) \\
& \Longrightarrow H_{*}^{c}\left(\left(\mathbb{T} \ltimes_{C_{p}} B^{\wedge p}\right)^{t C_{p}}\right) .
\end{aligned}
$$

It is not difficult to determine the $d^{2}$-differentials, and to prove that this spectral sequence collapses at the $\widehat{E}^{3}$-term, but we shall not need this result.

Recall the $C_{p}$-CW structure on $\mathbb{T}$ from Definition 3.8, which descends to a $\mathrm{CW}$ structure on the orbit space (= quotient group) $\mathbb{T} / C_{p}$, with $\bmod p$ cellular complex $C_{*}\left(\mathbb{T} / C_{p}\right)=\mathbb{F}_{p}\left\{e_{0}, e_{1}\right\}$ having boundary operator $d\left(e_{1}\right)=0$. Hence $H_{*}\left(\mathbb{T} / C_{p}\right)=\mathbb{F}_{p}\left\{e_{0}, e_{1}\right\}$, with $\operatorname{deg}\left(e_{j}\right)=j$ for $j \in\{0,1\}$.

Proposition 4.9. The map $\kappa$ induces the complete $\mathscr{A}_{*}$-comodule isomorphism

$$
\kappa_{*}: H_{*}\left(\mathbb{T} / C_{p}\right) \otimes H_{*}^{c}\left(\left(B^{\wedge p}\right)^{t C_{p}}\right) \stackrel{\cong}{\longrightarrow} H_{*}^{c}\left(\left(\mathbb{T} \ltimes_{C_{p}} B^{\wedge p}\right)^{t C_{p}}\right)
$$

given, modulo Tate filtration in the target, by

$$
e_{j} \otimes u^{i} t^{r} \otimes \alpha^{\otimes p} \longmapsto u^{i} t^{r} \otimes e_{j} \otimes \alpha^{\otimes p}
$$

for $(i, j)=(0,0),(1,0)$ or $(0,1)$, while

$$
\left(e_{1} \otimes u t^{r}-e_{0} \otimes t^{r}\right) \otimes \alpha^{\otimes p} \longmapsto u t^{r} \otimes e_{1} \otimes \alpha^{\otimes p}
$$

in the case $(i, j)=(1,1)$.

Remark 4.10. Note that acting by the fundamental class of $\mathbb{T} / C_{p}$ on classes represented in odd filtration has the effect of increasing the Tate filtration by 1, since the image of $e_{1} \otimes u t^{r} \otimes \alpha^{\otimes p}$ is represented by $t^{r} \otimes e_{0} \otimes \alpha^{\otimes p}$, modulo a term of lower Tate filtration.

Proof. For definiteness, we assume that our models for $E \mathbb{T}$ and $\widetilde{E T}$ are the unit sphere $S(\infty \mathbb{C})$ and the one-point compactification $S^{\infty \mathbb{C}}$ of $\infty \mathbb{C}=\mathbb{C}^{\infty}$ with the diagonal $\mathbb{T}$-action, respectively. We fix a based $\mathbb{T}$-CW structure on $\widetilde{E T}$ with $S^{n \mathbb{C}}$ as $\mathbb{T}$-equivariant $(2 n-1)$ - and $2 n$-skeleton, so that

$$
S^{n \mathbb{C}} / S^{(n-1) \mathbb{C}} \cong \Sigma^{2 n-1} \mathbb{T}_{+} .
$$

Restricting the action to $C_{p} \subset \mathbb{T}$, there is a based $C_{p^{-}} \mathrm{CW}$ structure on $\widetilde{E \mathbb{T}}$ with $S^{n \mathbb{C}}$ as $C_{p}$-equivariant $2 n$-skeleton, so that the subquotient $C_{p}$-CW structure on $S^{n \mathbb{C}} / S^{(n-1) \mathbb{C}}$ is the $(2 n-1)$-th suspension of the $C_{p}$-CW structure on $\mathbb{T}$ from Definition [3.8, based at a disjoint base point. For $n \geq 0$ let $\widetilde{E}_{n}$ be the $n$-skeleton of this based $C_{p}$-CW structure on $\widetilde{E T}$, viewed as a $C_{p}$-CW spectrum, and recall that the Greenlees filtration [17]

$$
\cdots \rightarrow \widetilde{E}_{n-1} \rightarrow \widetilde{E}_{n} \rightarrow \cdots \rightarrow \widetilde{E T}
$$

of $C_{p}$-spectra extends this notation to all integers $n$, so that $\widetilde{E}_{n} / \widetilde{E}_{n-1} \cong \Sigma^{n} C_{p+}$ and $\widetilde{E}_{2 n}=S^{n \mathbb{C}}$ for all $n \in \mathbb{Z}$. The cofiber sequence

$$
\widetilde{E}_{2 n-1} / \widetilde{E}_{2 n-2} \rightarrow \widetilde{E}_{2 n} / \widetilde{E}_{2 n-2} \rightarrow \widetilde{E}_{2 n} / \widetilde{E}_{2 n-1}
$$


equals the (2n-1)-th suspension of (4.3). If we only consider the even-indexed spectra, we get a coarser filtration

$$
\cdots \rightarrow \widetilde{E}_{2 n-2} \rightarrow \widetilde{E}_{2 n} \rightarrow \cdots \rightarrow \widetilde{E T}
$$

of $\mathbb{T}$-spectra, so that $\widetilde{E}_{2 n} / \widetilde{E}_{2 n-2} \cong \Sigma^{2 n-1} \mathbb{T}_{+}$for all $n$.

The homological Tate spectral sequence in Proposition 4.1 is obtained by expressing the $C_{p}$-Tate construction as a homotopy limit

$$
X^{t C_{p}} \simeq \operatorname{holim}_{n \rightarrow-\infty} X^{t C_{p}}[n]
$$

of a tower of spectra, where

$$
X^{t C_{p}}[n]=\left[\widetilde{E \mathbb{T}} / \widetilde{E}_{n-1} \wedge F\left(E \mathbb{T}_{+}, X\right)\right]^{C_{p}} .
$$

There are homotopy (co-)fiber sequences

$$
\left[\widetilde{E}_{n} / \widetilde{E}_{n-1} \wedge F\left(E \mathbb{T}_{+}, X\right)\right]^{C_{p}} \rightarrow X^{t C_{p}}[n] \rightarrow X^{t C_{p}}[n+1]
$$

and equivalences

$$
\begin{aligned}
{\left[\widetilde{E}_{n} / \widetilde{E}_{n-1} \wedge F\left(E \mathbb{T}_{+}, X\right)\right]^{C_{p}} } & \simeq\left[\widetilde{E}_{n} / \widetilde{E}_{n-1} \wedge X\right]^{C_{p}} \\
& \simeq\left(\widetilde{E}_{n} / \widetilde{E}_{n-1} \wedge X\right) / C_{p} \cong \Sigma^{n} X
\end{aligned}
$$

for all $n$, since $\widetilde{E}_{n} / \widetilde{E}_{n-1}$ is $C_{p}$-free. (Thus uses the Adams transfer equivalence $E / C_{p} \simeq E^{C_{p}}$ for $C_{p}$-free spectra $E$, see e.g. [21, §II.2].) The spectral sequence in question is associated to the exact couple with $A_{n, *}=H_{n+*}\left(X^{t C_{p}}[n]\right)$ and

$$
\widehat{E}_{n, *}^{1}=H_{n+*}\left(\left(\widetilde{E}_{n} / \widetilde{E}_{n-1} \wedge X\right) / C_{p}\right) \cong H_{*}(X) .
$$

In our case of interest, $X=\mathbb{T} \ltimes_{C_{p}} B^{\wedge p}$ is a $\mathbb{T}$-equivariant spectrum, and we seek to understand the residual $\mathbb{T} / C_{p}$-action on $X^{t C_{p}}$. The group $\mathbb{T}$ acts (only) on the even-indexed terms in the Greenlees filtration, hence $\mathbb{T} / C_{p}$ acts (only) on the odd-indexed terms in the homotopy limit above. Restricting to this coarser tower of spectra, we can express the $C_{p}$-Tate construction as the homotopy limit

$$
X^{t C_{p}} \simeq \operatorname{holim}_{n \rightarrow-\infty} X^{t C_{p}}[2 n-1]
$$

of $\mathbb{T} / C_{p}$-equivariant spectra. There are $\mathbb{T} / C_{p}$-equivariant homotopy (co-)fiber sequences

$$
\left[\widetilde{E}_{2 n} / \widetilde{E}_{2 n-2} \wedge F\left(E \mathbb{T}_{+}, X\right)\right]^{C_{p}} \rightarrow X^{t C_{p}}[2 n-1] \rightarrow X^{t C_{p}}[2 n+1]
$$

and equivalences

$$
\begin{aligned}
{\left[\widetilde{E}_{2 n} / \widetilde{E}_{2 n-2} \wedge F\left(E \mathbb{T}_{+}, X\right)\right]^{C_{p}} } & \simeq\left[\widetilde{E}_{2 n} / \widetilde{E}_{2 n-2} \wedge X\right]^{C_{p}} \\
& \simeq\left(\widetilde{E}_{2 n} / \widetilde{E}_{2 n-2} \wedge X\right) / C_{p} \cong \Sigma^{2 n-1} \mathbb{T}_{+} \wedge_{C_{p}} X
\end{aligned}
$$

for all $n$, since $\widetilde{E}_{2 n} / \widetilde{E}_{2 n-2}$ is $C_{p}$-free.

The map $\kappa$ is then realized as the homotopy limit of a tower of maps

$$
\kappa[2 n-1]: \mathbb{T} / C_{p} \ltimes\left(B^{\wedge p}\right)^{t C_{p}}[2 n-1] \rightarrow\left(\mathbb{T} \ltimes_{C_{p}} B^{\wedge p}\right)^{t C_{p}}[2 n-1]
$$

induced by the $C_{p}$-equivariant inclusion $i_{p}: B^{\wedge p} \rightarrow \mathbb{T} \ltimes_{C_{p}} B^{\wedge p}$ and the $\mathbb{T} / C_{p}$-action on the displayed target. Passing to homotopy fibers as in (4.6), we see that $\kappa$ in Tate filtrations $(2 n-1)$ and $2 n$ is represented by the map

$$
\bar{\kappa}: \mathbb{T} / C_{p} \ltimes\left(\Sigma^{2 n-1} \mathbb{T}_{+} \wedge_{C_{p}} B^{\wedge p}\right) \longrightarrow \Sigma^{2 n-1} \mathbb{T}_{+} \wedge_{C_{p}}\left(\mathbb{T} \ltimes_{C_{p}} B^{\wedge p}\right)
$$

induced by id $\wedge_{C_{p}} i_{p}$ and the $\mathbb{T} / C_{p}$-action. More explicitly, this is the map

$$
\bar{\kappa}=\Sigma^{2 n-1}\left(\xi \ltimes_{C_{p}} \text { id }\right)
$$

induced by the $C_{p}$-equivariant homeomorphism

$$
\xi: \mathbb{T} / C_{p} \times \mathbb{T} \stackrel{\cong}{\longrightarrow} \mathbb{T} \times_{C_{p}} \mathbb{T}
$$

that takes $([z], w)$ to $[z w, z]$. Here $z, w \in \mathbb{T}$ and square brackets indicate $C_{p}$-orbits.

A cellular approximation to $\xi$ induces the $C_{p}$-equivariant chain isomorphism

$$
\xi_{*}: C_{*}\left(\mathbb{T} / C_{p}\right) \otimes C_{*}(\mathbb{T}) \stackrel{\cong}{\longrightarrow} C_{*}(\mathbb{T}) \otimes_{C_{p}} C_{*}(\mathbb{T})
$$

given by

$$
e_{j} \otimes e_{k} \longmapsto e_{k} \otimes e_{j}
$$


for $(j, k)=(0,0),(0,1)$ or $(1,1)$, while

$$
e_{1} \otimes e_{0} \longmapsto e_{0} \otimes e_{1}+e_{1} \otimes T e_{0}
$$

in the case $(j, k)=(1,0)$. This follows by combining the cellular model $e_{0} \mapsto e_{0}, e_{1} \mapsto e_{0} \otimes e_{1}+e_{1} \otimes T e_{0}$ for the diagonal map $z \mapsto(z, z)$ with the cellular model $e_{j} \otimes e_{k} \mapsto e_{j+k}$ for $j+k \leq 1, e_{1} \otimes e_{1} \mapsto 0$ for the multiplication map $(z, w) \mapsto z w$.

Recall also that we made a choice of a chain equivalence $H_{*}(B) \simeq C_{*}(B)$, inducing a chain equivalence

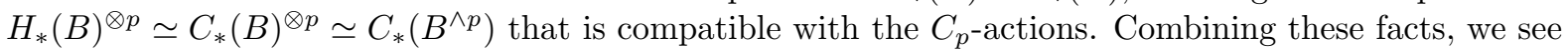
that $\bar{\kappa}$ has a chain level model

$$
\bar{\kappa}_{*}: C_{*}\left(\mathbb{T} / C_{p}\right) \otimes \Sigma^{2 n-1} C_{*}(\mathbb{T}) \otimes_{C_{p}} H_{*}(B)^{\otimes p} \stackrel{\cong}{\longrightarrow} \Sigma^{2 n-1} C_{*}(\mathbb{T}) \otimes_{C_{p}} C_{*}(\mathbb{T}) \otimes_{C_{p}} H_{*}(B)^{\otimes p}
$$

given by

$$
e_{j} \otimes \Sigma^{2 n-1} e_{k} \otimes x \longmapsto \Sigma^{2 n-1} e_{k} \otimes e_{j} \otimes x
$$

for $(j, k)=(0,0),(0,1)$ or $(1,1)$, and

$$
e_{1} \otimes \Sigma^{2 n-1} e_{0} \otimes x \longmapsto\left(\Sigma^{2 n-1} e_{0} \otimes e_{1}+\Sigma^{2 n-1} e_{1} \otimes T e_{0}\right) \otimes x
$$

in the case $(j, k)=(1,0)$, where $x \in H_{*}(B)^{\otimes p}$. Note that, as a consequence of linearity,

$$
e_{1} \otimes \Sigma^{2 n-1} e_{0} \otimes x-e_{0} \otimes \Sigma^{2 n-1} e_{1} \otimes T x \longmapsto \Sigma^{2 n-1} e_{0} \otimes e_{1} \otimes x .
$$

Now consider a class $u^{i} t^{r} \otimes \alpha^{\otimes p}$ in $H_{*}^{c}\left(\left(B^{\wedge p}\right)^{t C_{p}}\right)$. Let $n=-r$ and $k=1-i$. Then $u^{i} t^{r} \otimes \alpha^{\otimes p}$ lies in Tate filtration $-(i+2 r)=2 n-1+k$, and is represented by the homology class of

$$
\Sigma^{2 n-1} e_{k} \otimes \alpha^{\otimes p}
$$

in $H_{*}\left(\Sigma^{2 n-1} \mathbb{T}_{+} \wedge_{C_{p}} B^{\wedge p}\right)$. Hence $e_{j} \otimes u^{i} t^{r} \otimes \alpha^{\otimes p}$ is represented by the class of

$$
e_{j} \otimes \Sigma^{2 n-1} e_{k} \otimes \alpha^{\otimes p}
$$

in $H_{*}\left(\mathbb{T} / C_{p} \ltimes\left(\Sigma^{2 n-1} \mathbb{T}_{+} \wedge_{C_{p}} B^{\wedge p}\right)\right)$.

Its image under $\bar{\kappa}_{*}$ is given by the formulas (4.7) and (4.8) above, with $x=\alpha^{\otimes p}$, hence equals the class of

$$
\Sigma^{2 n-1} e_{k} \otimes e_{j} \otimes \alpha^{\otimes p}
$$

in $H_{*}\left(\Sigma^{2 n-1} \mathbb{T}_{+} \wedge_{C_{p}}\left(\mathbb{T} \ltimes_{C_{p}} B^{\wedge p}\right)\right)$ for $(j, k)=(0,0),(0,1)$ or $(1,1)$, and the class of

$$
\left(\Sigma^{2 n-1} e_{0} \otimes e_{1}+\Sigma^{2 n-1} e_{1} \otimes e_{0}\right) \otimes \alpha^{\otimes p}
$$

for $(j, k)=(1,0)$, since $T\left(\alpha^{\otimes p}\right)=\alpha^{\otimes p}$. It also follows that

$$
\left(e_{1} \otimes u t^{r}-e_{0} \otimes t^{r}\right) \otimes \alpha^{\otimes p}
$$

is represented by

which maps under $\bar{\kappa}_{*}$ to $\Sigma^{2 n-1} e_{0} \otimes e_{1} \otimes \alpha^{\otimes p}$.

$$
\left(e_{1} \otimes \Sigma^{2 n-1} e_{0}-e_{0} \otimes \Sigma^{2 n-1} e_{1}\right) \otimes \alpha^{\otimes p},
$$

To find the Tate representative of $\kappa_{*}\left(e_{j} \otimes u^{i} t^{r} \otimes \alpha^{\otimes p}\right)$ we now use the cofiber sequence (4.4) and the associated cofiber sequence

$$
\Sigma^{2 n-1}\left(\mathbb{T} \ltimes_{C_{p}} B^{\wedge p}\right) \rightarrow \Sigma^{2 n-1} \mathbb{T}_{+} \wedge_{C_{p}}\left(\mathbb{T} \ltimes_{C_{p}} B^{\wedge p}\right) \rightarrow \Sigma^{2 n}\left(\mathbb{T} \ltimes_{C_{p}} B^{\wedge p}\right)
$$

coming from the Tate filtration of the middle term. It shows that $\Sigma^{2 n-1} e_{k} \otimes e_{j} \otimes \alpha^{\otimes p}$ in

$$
H_{*}\left(\Sigma^{2 n-1} \mathbb{T}_{+} \wedge_{C_{p}}\left(\mathbb{T} \ltimes_{C_{p}} B^{\wedge p}\right)\right)
$$

has Tate representative $u^{i} t^{r} \otimes e_{j} \otimes \alpha^{\otimes p}$, where $i=1-k$ and $r=-n$. Noting that the case $(i, j)=(1,1)$ corresponds to the case $(j, k)=(1,0)$, and chasing the formulas, we get the asserted result.

Theorem 4.11. The map

$$
\omega^{t}: \mathbb{T} / C_{p} \ltimes\left(B^{\wedge p}\right)^{t C_{p}} \longrightarrow \mathrm{THH}(B)^{t C_{p}}
$$

induces the complete $\mathscr{A}_{*}$-comodule homomorphism

$$
\omega_{*}^{t}: H_{*}\left(\mathbb{T} / C_{p}\right) \otimes H_{*}^{c}\left(\left(B^{\wedge p}\right)^{t C_{p}}\right) \longrightarrow H_{*}^{c}\left(\operatorname{THH}(B)^{t C_{p}}\right)
$$

given, modulo Tate filtration in the target, by

$$
\begin{aligned}
e_{0} \otimes u^{i} t^{r} \otimes \alpha^{\otimes p} & \longmapsto u^{i} t^{r} \otimes \alpha^{p} \\
e_{1} \otimes t^{r} \otimes \alpha^{\otimes p} & \longmapsto t^{r} \otimes \alpha^{p-1} \wedge \alpha \\
\left(e_{1} \otimes u t^{r}-e_{0} \otimes t^{r}\right) \otimes \alpha^{\otimes p} & \longmapsto u t^{r} \otimes \alpha^{p-1} \wedge \alpha
\end{aligned}
$$


for $i \in\{0,1\}, r \in \mathbb{Z}$.

Proof. This is clear from Proposition 4.9, Definition 3.10, and naturality of the homological Tate spectral sequence with respect to the map $D \circ \omega_{p}: \mathbb{T} \ltimes_{C_{p}} B^{\wedge p} \rightarrow \mathrm{THH}(B)$.

\section{Cyclotomic structure}

Recall from diagram (2.1) that we are interested in the natural map

$$
\left.\hat{\Gamma}:[\widetilde{E T} \wedge X]^{C_{p}} \rightarrow \widetilde{E \mathbb{T}} \wedge F\left(E \mathbb{T}_{+}, X\right)\right]^{C_{p}}=X^{t C_{p}}
$$

for $X \cong \mathrm{THH}(B)$. We now study the source of this map. This allows us to construct the commutative diagram (2.4), which appears in Theorem 5.3 .

Definition 5.1. Let $G \subseteq \mathbb{T}$ be a closed subgroup containing $C_{p}$. For each good $G$-equivariant prespectrum $Y$, with spectrification $X=L Y$, the geometric fixed point prespectrum $\Phi^{C_{p}}(Y)$ is the $G / C_{p^{-}}$ equivariant prespectrum with

$$
\Phi^{C_{p}}(Y)\left(V^{C_{p}}\right)=Y(V)^{C_{p}},
$$

and the geometric fixed point spectrum $\Phi^{C_{p}}(X)$ is its spectrification $\Phi^{C_{p}}(X)=L \Phi^{C_{p}}(Y)$. See [21, II.9.7] and [19, §2.1].

There is a natural equivalence of $G / C_{p}$-spectra

$$
\bar{s}:[\widetilde{E T} \wedge X]^{C_{p}} \stackrel{\simeq}{\longrightarrow} \Phi^{C_{p}}(X),
$$

see [21, II.9.8] and the proof of [19, Lem. 2.1]. We are concerned with the cases $G=C_{p}, Y=\left(B_{\mathrm{pre}}^{\wedge p}\right)^{\tau}$ and $G=\mathbb{T}, Y=\operatorname{sd}_{p} \operatorname{thh}(B)^{\tau}$, corresponding to $X=B^{\wedge p}$ and $X=\operatorname{sd}_{p} \operatorname{THH}(B)$, respectively.

With notation as in Definition 3.1, there are natural isomorphisms and maps

$$
\begin{aligned}
& \operatorname{sd}_{p} \operatorname{thh}\left(B ; S^{V}\right)_{k}^{C_{p}}=\operatorname{thh}\left(B ; S^{V}\right)_{p(k+1)-1}^{C_{p}} \\
& =\left(\underset{\overrightarrow{\mathbf{n}} \in I^{p(k+1)}}{\operatorname{hocolim}} \operatorname{Map}\left(S^{n_{0}} \wedge \cdots \wedge S^{n_{p(k+1)-1}}, B_{n_{0}} \wedge \cdots \wedge B_{n_{p(k+1)-1}} \wedge S^{V}\right)\right)^{C_{p}} \\
& \cong \underset{\overrightarrow{\mathbf{n}} \in I^{k+1}}{\operatorname{hocolim} \operatorname{Map}}\left(\left(S^{n_{0}} \wedge \cdots \wedge S^{n_{k}}\right)^{\wedge p},\left(B_{n_{0}} \wedge \cdots \wedge B_{n_{k}}\right)^{\wedge p} \wedge S^{V}\right)^{C_{p}} \\
& \rightarrow \underset{\overrightarrow{\mathbf{n}} \in I^{k+1}}{\operatorname{hocolim}} \operatorname{Map}\left(S^{n_{0}} \wedge \cdots \wedge S^{n_{k}}, B_{n_{0}} \wedge \cdots \wedge B_{n_{k}} \wedge S^{V^{C_{p}}}\right)=\operatorname{thh}\left(B ; S^{V^{C_{p}}}\right)_{k}
\end{aligned}
$$

induced from the identifications $S^{n_{0}} \wedge \cdots \wedge S^{n_{k}} \cong\left(\left(S^{n_{0}} \wedge \cdots \wedge S^{n_{k}}\right)^{\wedge p}\right)^{C_{p}}$ and $B_{n_{0}} \wedge \cdots \wedge B_{n_{k}} \cong$ $\left(\left(B_{n_{0}} \wedge \cdots \wedge B_{n_{k}}\right)^{\wedge p}\right)^{C_{p}}$. These define a natural map of prespectra

$$
r_{k}^{\prime}: \Phi^{C_{p}}\left(\operatorname{sd}_{p} \operatorname{thh}(B)_{k}\right) \rightarrow \operatorname{thh}(B)_{k}
$$

for each $k \geq 0$, and likewise after the natural good thickening.

Lemma 5.2. Suppose that $B$ is connective. The spectrum maps

$$
r_{0}^{\prime}: \Phi^{C_{p}}\left(B^{\wedge p}\right) \stackrel{\simeq}{\longrightarrow} B^{\wedge 1}
$$

(corresponding to the case $k=0$ ) and

$$
r^{\prime}: \Phi^{C_{p}}\left(\operatorname{sd}_{p} \operatorname{THH}(B)\right) \stackrel{\simeq}{\longrightarrow} \mathrm{THH}(B)
$$

(obtained from the cases $k \geq 0$ by geometric realization) are natural equivalences.

Proof. The second case is proved in [19, Prop. 2.5], and the first case is part of their proof. In more detail, they prove that the connectivity of the map

$$
\Omega^{V^{C_{p}}-W} r_{0}^{\prime}\left(V^{C_{p}}\right): \Omega^{V^{C_{p}}-W} \operatorname{thh}\left(B ; S^{V}\right)_{p-1}^{C_{p}} \longrightarrow \Omega^{V^{C_{p}}-W} \operatorname{thh}\left(B ; S^{V^{C_{p}}}\right)_{0}
$$

grows to infinity with $V$, for each fixed $W$. (The connectivity hypothesis enters at the bottom of page 42 in the cited paper.)

In the case $G=\mathbb{T}$, let $\rho: \mathbb{T} \rightarrow \mathbb{T} / C_{p}$ be the $p$-th root isomorphism of groups, with inverse $\rho^{-1}: \mathbb{T} / C_{p} \rightarrow$ $\mathbb{T}$ taking $[z]$ to $z^{p}$. The cyclic structures on $\operatorname{sd}_{p} \operatorname{thh}(B)$ • and $\operatorname{thh}(B)$ • induce a $\mathbb{T} / C_{p}$-equivariant structure on $\Phi^{C_{p}}\left(\operatorname{sd}_{p} \operatorname{THH}(B)\right)$ and a $\mathbb{T}$-equivariant structure on $\operatorname{THH}(B)$. These are compatible, in the sense that the cyclotomic structure equivalence $r^{\prime}$ is $\rho^{-1}$-equivariant. See [19, Def. 2.2]. 


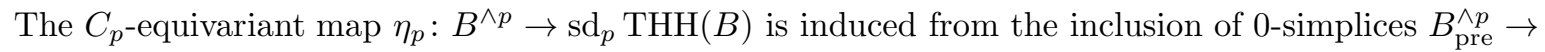
$\operatorname{sd}_{p} \operatorname{thh}(B) \bullet$, hence is compatible with $\bar{s}$ by naturality, and with the maps $r_{0}^{\prime}$ and $r^{\prime}$ by the construction of the latter via geometric realization. Hence the outer part of the following diagram commutes.

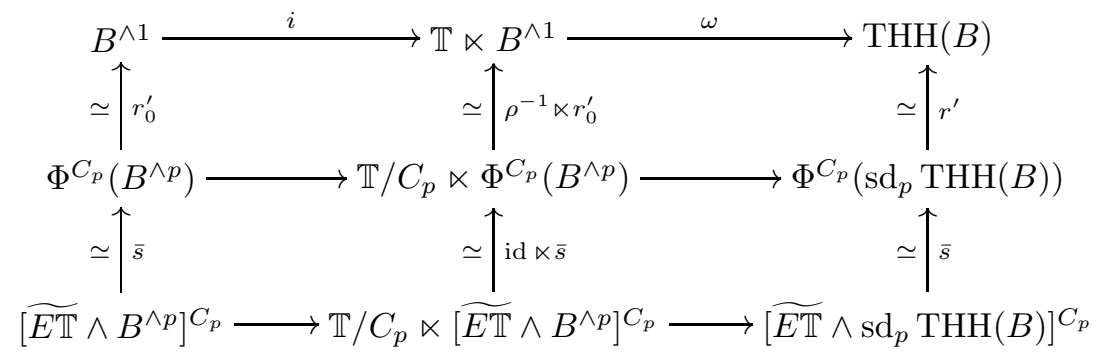

The right hand horizontal maps are the unique equivariant extensions, given by the $\mathbb{T} / C_{p}$-action on $[\widetilde{E T} \wedge$ $\left.\operatorname{sd}_{p} \operatorname{THH}(B)\right]^{C_{p}}$ and $\Phi^{C_{p}}\left(\operatorname{sd}_{p} \operatorname{THH}(B)\right)$, and the $\mathbb{T}$-action on $\mathrm{THH}(B)$, respectively. These equivariant extensions are compatible, via the identity id: $\mathbb{T} / C_{p} \rightarrow \mathbb{T} / C_{p}$ and the isomorphism $\rho^{-1}: \mathbb{T} / C_{p} \rightarrow \mathbb{T}$, respectively, in view of the equivariance properties of $\bar{s}$ and $r^{\prime}$ discussed above. Hence the whole diagram commutes.

Theorem 5.3. Let $B$ be a connective symmetric ring spectrum. There is a natural commutative diagram in the stable homotopy category

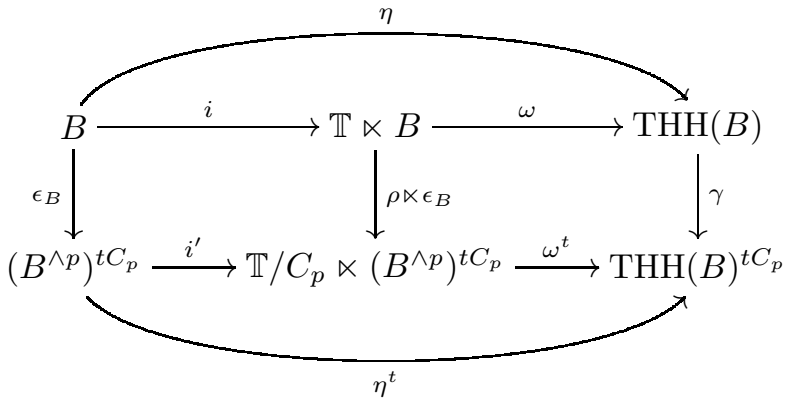

where $\epsilon_{B}$ is the composite

$$
\left.B \stackrel{\simeq}{\longleftarrow} \widetilde{E T} \wedge B^{\wedge p}\right]^{C_{p}} \stackrel{\hat{\Gamma}}{\longrightarrow}\left(B^{\wedge p}\right)^{t C_{p}}=R_{+}(B)
$$

and $\gamma$ is the composite

$$
\left.\operatorname{THH}(B) \stackrel{\simeq}{\longleftarrow} \widetilde{E T} \wedge \operatorname{sd}_{p} \operatorname{THH}(B)\right]^{C_{p}} \stackrel{\hat{\Gamma}}{\longrightarrow}\left(\operatorname{sd}_{p} \operatorname{THH}(B)\right)^{t C_{p}} \cong \operatorname{THH}(B)^{t C_{p}}
$$

Proof. By naturality of the map $\hat{\Gamma}$ with respect to the inclusion of 0 -simplices $\eta_{p}: B^{\wedge p} \rightarrow \operatorname{sd}_{p} \operatorname{THH}(B)$ we get that the outer part of the following diagram commutes.

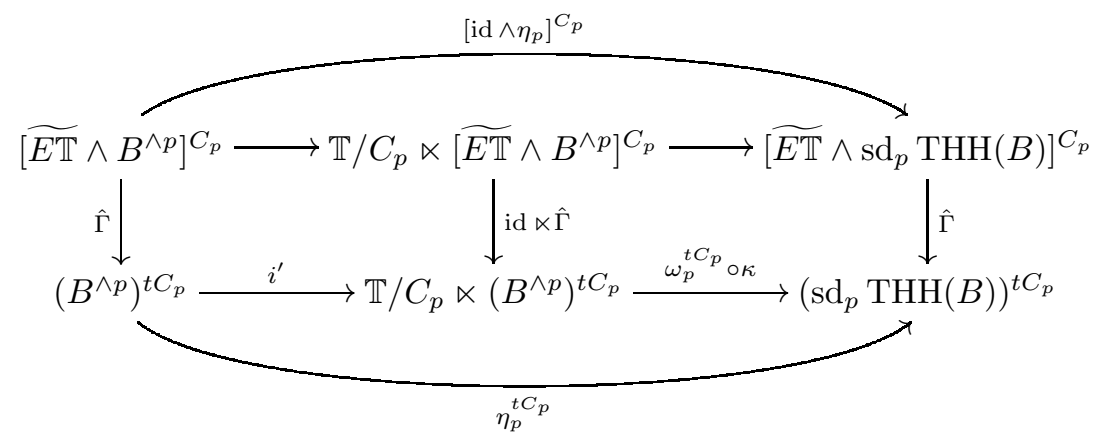

The right hand column is $\mathbb{T} / C_{p}$-equivariant, and the right hand square is constructed to be the unique $\mathbb{T} / C_{p}$-equivariant extension of the outer part. Hence the whole diagram commutes. 
By combining the right hand parts of diagrams (5.1), (5.2) and (4.2), we get the central part of the commutative diagram below:

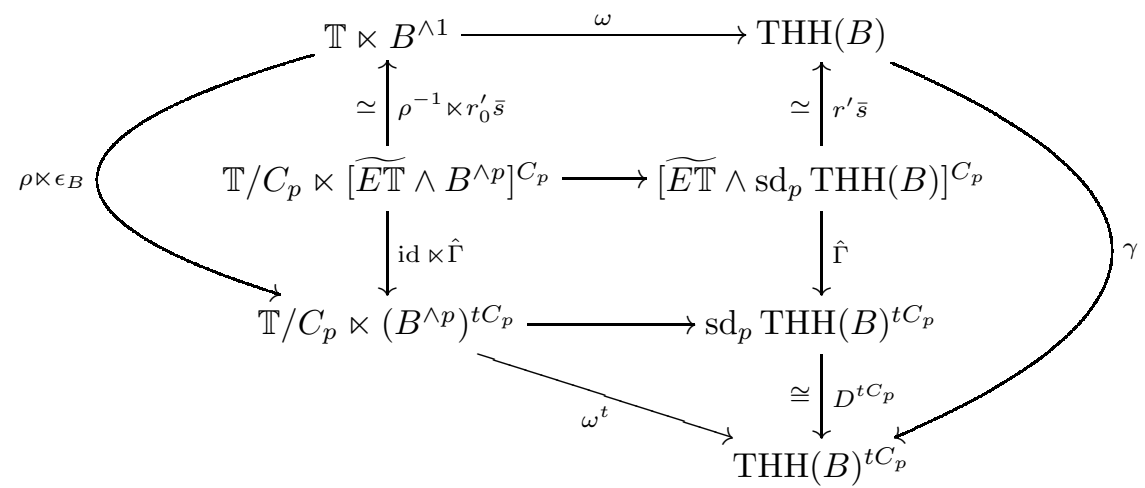

This proves the theorem.

\section{The COMPARISON MAP}

In this section we compute the effect of the comparison map $\gamma: \mathrm{THH}(B) \rightarrow \mathrm{THH}(B)^{t C_{p}}$ for $B=M U$ and $B P$. The main results are Theorems 6.4 and 6.9.

Let $H=H \mathbb{F}_{p}$ be the $\bmod p$ Eilenberg-Mac Lane spectrum, realized as a commutative symmetric ring spectrum. Recall [26] the structure

$$
\mathscr{A}_{*}=H_{*}(H)=P\left(\bar{\xi}_{k} \mid k \geq 1\right) \otimes E\left(\bar{\tau}_{k} \mid k \geq 0\right)
$$

of the dual Steenrod algebra, where $\left|\bar{\xi}_{k}\right|=2 p^{k}-2$ and $\left|\bar{\tau}_{k}\right|=2 p^{k}-1$. (This assumes $p$ is odd-we leave the details for $p=2$ to the reader.) The Hopf algebra coproduct is given by the formulas

$$
\begin{aligned}
\psi\left(\bar{\xi}_{k}\right) & =\sum_{i+j=k} \bar{\xi}_{i} \otimes \bar{\xi}_{j}^{p^{i}} \\
\psi\left(\bar{\tau}_{k}\right) & =1 \otimes \bar{\tau}_{k}+\sum_{i+j=k} \bar{\tau}_{i} \otimes \bar{\xi}_{j}^{p^{i}}
\end{aligned}
$$

where $\bar{\xi}_{0}=1$.

6.1. The case of complex cobordism. Let $M U$ be the complex cobordism spectrum, realized as a commutative symmetric ring spectrum [24, IV.2]. Recall [1, pp. 75-77] the $\mathscr{A}_{*}$-comodule algebra isomorphism

$$
H_{*}(M U) \cong P\left(\bar{\xi}_{k} \mid k \geq 1\right) \otimes P\left(m_{\ell} \mid \ell \neq p^{k}-1\right),
$$

where $m_{\ell}$ is an $\mathscr{A}_{*}$-comodule primitive of degree $2 \ell$, for each $\ell \geq 1$ not of the form $p^{k}-1$. Note that $H_{*}(M U)$ is concentrated in even degrees.

Definition 6.1. For $\ell=p^{k}-1$, let $m_{\ell}=\bar{\xi}_{k}$, so that $H_{*}(M U) \cong P\left(m_{\ell} \mid \ell \geq 1\right)$.

Lemma 6.2. There is an $\mathscr{A}_{*}$-comodule algebra isomorphism

$$
H_{*}(\mathrm{THH}(M U)) \cong H_{*}(M U) \otimes E\left(\sigma m_{\ell} \mid \ell \geq 1\right) .
$$

The classes $\sigma m_{\ell}$ are $\mathscr{A}_{*}$-comodule primitive, for all $\ell \geq 1$.

Proof. We use the (first quadrant) Bökstedt spectral sequence

$$
E_{*, *}^{2}=H H_{*}\left(H_{*}(B)\right) \Longrightarrow H_{*}(\mathrm{THH}(B))
$$

arising from the skeleton filtration on $\operatorname{THH}(B)$, i.e., the filtration induced from the simplicial structure on $\operatorname{thh}(B)$. See [3, §5] for the tools used in this computation, including the facts that $\sigma$ is a derivation, and that it commutes with the Dyer-Lashof operations $Q^{i}$.

The $E^{2}$-term for $B=M U$ is

$$
E_{*, *}^{2}=H_{*}(M U) \otimes E\left(\sigma m_{\ell} \mid \ell \geq 1\right) .
$$

We have $E_{*, *}^{2}=E_{*, *}^{\infty}$, since all algebra generators lie in filtrations $\leq 1$. There are no algebra extensions, since $\sigma m_{\ell}$ is in an odd degree, hence is an exterior class for $p$ odd by graded commutativity, and for $p=2$ by the Dyer-Lashof calculation $\left(\sigma m_{\ell}\right)^{2}=Q^{2 \ell+1}\left(\sigma m_{\ell}\right)=\sigma Q^{2 \ell+1}\left(m_{\ell}\right)=0$, since $Q^{2 \ell+1}\left(m_{\ell}\right) \in$ 
$H_{4 \ell+1}(M U)=0$. The $\mathscr{A}_{*}$-coaction $\nu: H_{*}(X) \rightarrow \mathscr{A}_{*} \otimes H_{*}(X)$ for $X=\operatorname{THH}(M U)$ is given by $\nu\left(\sigma m_{\ell}\right)=$ $(1 \otimes \sigma) \nu\left(m_{\ell}\right)=(1 \otimes \sigma)\left(1 \otimes m_{\ell}\right)=1 \otimes \sigma m_{\ell}$ for $\ell \neq p^{k}-1$, while

$$
\nu\left(\sigma \bar{\xi}_{k}\right)=(1 \otimes \sigma)\left(\sum_{i+j=k} \bar{\xi}_{i} \otimes \bar{\xi}_{j}^{p^{i}}\right)=\sum_{i+j=k} \bar{\xi}_{i} \otimes \sigma\left(\bar{\xi}_{j}^{p^{i}}\right)=1 \otimes \sigma \bar{\xi}_{k},
$$

since $\sigma\left(\bar{\xi}_{j}^{p^{i}}\right)=0$ for $i \geq 1$.

The following was proved by a different method in [14, 6.4].

Proposition 6.3. The homological Tate spectral sequence

$$
\widehat{E}_{*, *}^{2}(\operatorname{THH}(M U))=\widehat{H}^{-*}\left(C_{p} ; H_{*}(\operatorname{THH}(M U))\right) \Longrightarrow H_{*}^{c}\left(\operatorname{THH}(M U)^{t C_{p}}\right)
$$

collapses at the $\widehat{E}^{3}=\widehat{E}^{\infty}$-term, with

$$
\widehat{E}_{*, *}^{\infty}=\widehat{H}^{-*}\left(C_{p} ; \mathbb{F}_{p}\right) \otimes P\left(m_{\ell}^{p} \mid \ell \geq 1\right) \otimes E\left(m_{\ell}^{p-1} \sigma m_{\ell} \mid \ell \geq 1\right) .
$$

Proof. The $\widehat{E}^{2}$-term is

$$
\widehat{E}_{*, *}^{2}=\widehat{H}^{-*}\left(C_{p} ; \mathbb{F}_{p}\right) \otimes P\left(m_{\ell} \mid \ell \geq 1\right) \otimes E\left(\sigma m_{\ell} \mid \ell \geq 1\right)
$$

where $\widehat{H}^{-*}\left(C_{p} ; \mathbb{F}_{p}\right)=E(u) \otimes P\left(t, t^{-1}\right)$ for $p$ odd. The classes $u^{i} t^{r}$ are all infinite cycles, as recalled in Remark 4.3. The $d^{2}$-differentials are given by the formula

$$
d^{2}\left(u^{i} t^{r} \otimes \alpha\right)=u^{i} t^{r+1} \otimes \sigma \alpha,
$$

see e.g. [14, 3.2]. The homology of $P\left(m_{\ell}\right) \otimes E\left(\sigma m_{\ell}\right)$ with respect to $\sigma$ is $P\left(m_{\ell}^{p}\right) \otimes E\left(m_{\ell}^{p-1} \sigma m_{\ell}\right)$, so by the Künneth formula

$$
\widehat{E}_{*, *}^{3}=\widehat{H}^{-*}\left(C_{p} ; \mathbb{F}_{p}\right) \otimes P\left(m_{\ell}^{p} \mid \ell \geq 1\right) \otimes E\left(m_{\ell}^{p-1} \sigma m_{\ell} \mid \ell \geq 1\right) .
$$

By Theorem 4.11 and Corollary 3.13 the map

$$
\omega^{t}: \mathbb{T} / C_{p} \ltimes\left(B^{\wedge p}\right)^{t C_{p}} \rightarrow \mathrm{THH}(B)^{t C_{p}}
$$

for $B=M U$ takes the classes $e_{0} \otimes 1 \otimes m_{\ell}^{\otimes p}$ and $e_{1} \otimes 1 \otimes m_{\ell}^{\otimes p}$ in $H_{*}\left(\mathbb{T} / C_{p}\right) \otimes H_{*}^{c}\left(\left(B^{\wedge p}\right)^{t C_{p}}\right)$ to classes represented by $1 \otimes m_{\ell}^{p}$ and $1 \otimes m_{\ell}^{p-1} \sigma m_{\ell}$ in the Tate spectral sequence, respectively. Hence the $\widehat{E}^{3}$-term is generated by infinite cycles, and there cannot be any further differentials.

Theorem 6.4. The map

$$
\gamma: \operatorname{THH}(M U) \rightarrow \operatorname{THH}(M U)^{t C_{p}}
$$

induces a complete $\mathscr{A}_{*}$-comodule algebra homomorphism

$$
\gamma_{*}: H_{*}(\mathrm{THH}(M U)) \longrightarrow H_{*}^{c}\left(\mathrm{THH}(M U)^{t C_{p}}\right)
$$

mapping

$$
\sigma m_{\ell} \longmapsto(-1)^{\ell} t^{(p-1) \ell} \otimes m_{\ell}^{p-1} \sigma m_{\ell}
$$

modulo Tate filtration in the target, for each $\ell \geq 1$.

Proof. We use the commutative diagram

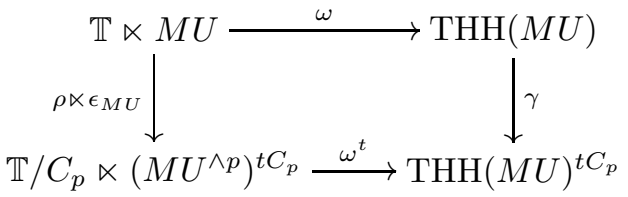

from Theorem $\left[5.3\right.$ for $B=M U$. Let $[\mathbb{T}] \in H_{1}(\mathbb{T})$ be the fundamental class, which maps by $\rho_{*}$ to the fundamental class $e_{1} \in H_{1}\left(\mathbb{T} / C_{p}\right)$. Then $\omega_{*}([\mathbb{T}] \otimes \alpha)=\sigma \alpha$ for all $\alpha \in H_{*}(M U)$, so we can compute $\gamma_{*}(\sigma \alpha)$ as the image under $\omega_{*}^{t}$ of $e_{1} \otimes\left(\epsilon_{M U}\right)_{*}(\alpha)$. We make separate calculations for the cases $\alpha=\bar{\xi}_{k}$ (with $\ell=p^{k}-1$ ) and $\alpha=m_{\ell}\left(\right.$ with $\left.\ell \neq p^{k}-1\right)$.

Recall from [23, 3.2.1] that the homomorphism

$$
\epsilon_{*}: H_{*}(M U) \rightarrow R_{+}\left(H_{*}(M U)\right)
$$

is given by the formula

$$
\epsilon_{*}(\alpha)=\sum_{r=0}^{\infty} t^{-(p-1) r} \otimes(-1)^{r} \mathrm{P}_{*}^{r}(\alpha)
$$


in the homological Singer construction $R_{+}\left(H_{*}(M U)\right)$, where $\mathrm{P}_{*}^{r}$ is the homology operation dual to the $r$-th Steenrod power. (The terms involving $\left(\beta \mathrm{P}^{r}\right)_{*}(\alpha)$ vanish in this case, since $H_{*}(M U)$ is concentrated in even degrees.)

By Lemma 6.5 below, we obtain

$$
\begin{aligned}
\epsilon_{*}\left(\bar{\xi}_{k}\right) & =\sum_{i=0}^{k} t^{-\left(p^{i}-1\right)} \otimes \bar{\xi}_{k-i}^{p^{i}} \\
& =1 \otimes \bar{\xi}_{k}+t^{-(p-1)} \otimes \bar{\xi}_{k-1}^{p}+\cdots+t^{-\left(p^{k}-1\right)} \otimes 1
\end{aligned}
$$

in $R_{+}\left(H_{*}(M U)\right)$, for each $k \geq 1$. To control the terms with $1 \leq i \leq k$, it will be convenient to compare with the $\epsilon_{*}$-image of $\bar{\xi}_{k-1}^{p}$. By Lemma 6.6 below, we obtain

$$
\epsilon_{*}\left(\bar{\xi}_{k-1}^{p}\right)=\sum_{i=0}^{k-1} t^{-p\left(p^{i}-1\right)} \otimes \bar{\xi}_{k-1-i}^{p^{i+1}}=\sum_{i=1}^{k} t^{-\left(p^{i}-p\right)} \otimes \bar{\xi}_{k-i}^{p^{i}} .
$$

Hence

$$
\epsilon_{*}\left(\bar{\xi}_{k}\right)=1 \otimes \bar{\xi}_{k}+t^{-(p-1)} \cdot \epsilon_{*}\left(\bar{\xi}_{k-1}^{p}\right)
$$

in $R_{+}\left(H_{*}(M U)\right)$, for each $k \geq 1$. Here the multiplication by $t^{-(p-1)}$ refers to the $R_{+}\left(H_{*}(S)\right)=$ $\widehat{H}^{-*}\left(C_{p} ; \mathbb{F}_{p}\right)$-module structure on $R_{+}\left(H_{*}(M U)\right)$, coming from the $S$-module structure on $M U$.

Next recall the isomorphism $R_{+}\left(H_{*}(M U)\right) \cong H_{*}^{c}\left(\left(M U^{\wedge p}\right)^{t C_{p}}\right)$ of [23, 5.14], taking $t^{r} \otimes \alpha$ to a preferred class represented by

$$
(-1)^{\ell} t^{r+(p-1) \ell} \otimes \alpha^{\otimes p}
$$

in the Tate spectral sequence, where $|\alpha|=2 \ell$ is assumed to be even. (The coefficient is more complicated when $|\alpha|$ is odd.) By [23, 5.12], the map $\epsilon_{M U}: M U \rightarrow\left(M U^{\wedge p}\right)^{t C_{p}}$ induces the composite of $\epsilon_{*}: H_{*}(M U) \rightarrow R_{+}\left(H_{*}(M U)\right)$ and this isomorphism. Hence, the identity (6.1) tells us that the difference

$$
\left(\epsilon_{M U}\right)_{*}\left(\bar{\xi}_{k}\right)-t^{-(p-1)} \cdot\left(\epsilon_{M U}\right)_{*}\left(\bar{\xi}_{k-1}^{p}\right)
$$

is the preferred class represented by

$$
t^{(p-1)\left(p^{k}-1\right)} \otimes \bar{\xi}_{k} \otimes p
$$

in the Tate spectral sequence converging to $H_{*}^{c}\left(\left(M U^{\wedge p}\right)^{t C_{p}}\right)$.

We now chase the class $[\mathbb{T}] \otimes \bar{\xi}_{k-1}^{p}$ in $H_{*}(\mathbb{T} \ltimes M U)$ around the commutative square above. Going to the right,

$$
\omega_{*}\left([\mathbb{T}] \otimes \bar{\xi}_{k-1}^{p}\right)=\sigma\left(\bar{\xi}_{k-1}^{p}\right)=0
$$

in $H_{*}(\mathrm{THH}(M U))$, since $\sigma$ is a derivation. Hence the image under $\gamma_{*}$ is also 0 , which implies that

$$
\left(\omega^{t}\right)_{*}\left(e_{1} \otimes\left(\epsilon_{M U}\right)_{*}\left(\bar{\xi}_{k-1}^{p}\right)\right)=0 .
$$

It follows by the $\widehat{H}^{-*}\left(C_{p} ; \mathbb{F}_{p}\right)$-module structure that

$$
\left(\omega^{t}\right)_{*}\left(e_{1} \otimes t^{-(p-1)} \cdot\left(\epsilon_{M U}\right)_{*}\left(\bar{\xi}_{k-1}^{p}\right)\right)=0 .
$$

Finally we chase the class $[\mathbb{T}] \otimes \bar{\xi}_{k}$ around the square, to see that $\gamma_{*}\left(\sigma \bar{\xi}_{k}\right)$ equals

$$
\left(\omega^{t}\right)_{*}\left(e_{1} \otimes\left(\epsilon_{M U}\right)_{*}\left(\bar{\xi}_{k}\right)\right)=\left(\omega^{t}\right)_{*}\left(e_{1} \otimes t^{(p-1)\left(p^{k}-1\right)} \otimes \bar{\xi}_{k}^{\otimes p}\right),
$$

which by Theorem 4.11 and Corollary 3.13 is represented by

$$
t^{(p-1)\left(p^{k}-1\right)} \otimes \bar{\xi}_{k}^{p-1} \sigma \bar{\xi}_{k}
$$

in the Tate spectral sequence converging to $H_{*}^{c}\left(\mathrm{THH}(M U)^{t C_{p}}\right)$. This proves the theorem for $m_{\ell}=\bar{\xi}_{k}$, with $\ell=p^{k}-1$, since $(-1)^{\ell} \equiv+1 \bmod p$ in these cases.

The remaining cases, of $m_{\ell}$ with $\ell \neq p^{k}-1$, are simpler. We have

$$
\epsilon_{*}\left(m_{\ell}\right)=1 \otimes m_{\ell}
$$

in the homological Singer construction, since these $m_{\ell}$ 's are $\mathscr{A}_{*}$-comodule primitives. Hence $\left(\epsilon_{M U}\right)_{*}\left(m_{\ell}\right)$ is the preferred class represented by

$$
(-1)^{\ell} t^{(p-1) \ell} \otimes m_{\ell} \otimes p
$$

in the Tate spectral sequence converging to $H_{*}^{c}\left(\left(M U^{\wedge p}\right)^{t C_{p}}\right)$. Chasing $[\mathbb{T}] \otimes m_{\ell}$ around the commutative diagram, we find that $\gamma_{*}\left(\sigma m_{\ell}\right)$ equals

$$
\omega_{*}^{t}\left(e_{1} \otimes(-1)^{\ell} t^{(p-1) \ell} \otimes m_{\ell} \otimes p\right)=(-1)^{\ell} t^{(p-1) \ell} \otimes m_{\ell}^{p-1} \sigma m_{\ell} .
$$




\section{Lemma 6.5.}

$$
(-1)^{r} \mathrm{P}_{*}^{r}\left(\bar{\xi}_{k}\right)= \begin{cases}\bar{\xi}_{k-i}^{p^{i}} & \text { if } r=\left(p^{i}-1\right) /(p-1), \\ 0 & \text { otherwise. }\end{cases}
$$

Proof. The Steenrod power $\mathrm{P}^{r}$ is dual to $\xi_{1}^{r}$ in the Milnor basis $\left(\xi^{I} \tau^{J}\right)$ for $\mathscr{A}_{*}$, so each term of the form $\xi_{1}^{r} \otimes \alpha^{\prime \prime}$ in the coaction $\nu(\alpha)$ contributes a term $\alpha^{\prime \prime}$ in $\mathrm{P}_{*}^{r}(\alpha)$. From the recursive relation $0=\sum_{i+j=k} \xi_{i}^{p^{j}} \cdot \bar{\xi}_{j}$ for $k \geq 1$, we see that $\bar{\xi}_{i} \equiv(-1)^{i} \xi_{1}^{\left(p^{i}-1\right) /(p-1)}$ for $i \geq 0$, modulo the ideal $J(0) \subset \mathscr{A}_{*}$ generated by the $\xi_{k}$ with $k \geq 2$ and the $\tau_{k}$ with $k \geq 1$. Hence $\nu\left(\bar{\xi}_{k}\right)=\sum_{i+j=k} \bar{\xi}_{i} \otimes \bar{\xi}_{j}^{p^{i}}$ is congruent to

$$
\sum_{i=0}^{k}(-1)^{i} \xi_{1}^{\left(p^{i}-1\right) /(p-1)} \otimes \bar{\xi}_{k-i}^{p^{i}}
$$

and contributes $(-1)^{i} \bar{\xi}_{k-i}^{p^{i}}$ to $\mathrm{P}_{*}^{r}\left(\bar{\xi}_{k}\right)$ precisely if $r=\left(p^{i}-1\right) /(p-1)$. In this case $(-1)^{i} \equiv(-1)^{r}$ $\bmod p$.

Lemma 6.6.

$$
(-1)^{r} \mathrm{P}_{*}^{r}\left(\bar{\xi}_{k-1}^{p}\right)= \begin{cases}\bar{\xi}_{k-1-i}^{p^{i+1}} & \text { if } r=p\left(p^{i}-1\right) /(p-1) \\ 0 & \text { otherwise }\end{cases}
$$

Proof. The coaction $\nu\left(\bar{\xi}_{k-1}^{p}\right)=\sum_{i+j=k-1} \bar{\xi}_{i}^{p} \otimes \bar{\xi}_{j}^{p^{i+1}}$ is congruent to

$$
\sum_{i=0}^{k-1}(-1)^{i} \xi_{1}^{p\left(p^{i}-1\right) /(p-1)} \otimes \bar{\xi}_{k-1-i}^{p^{i+1}},
$$

and contributes $(-1)^{i} \bar{\xi}_{k-1-i}^{p^{i+1}}$ to $\mathrm{P}_{*}^{r}\left(\bar{\xi}_{k-1}^{p}\right)$ precisely if $r=p\left(p^{i}-1\right) /(p-1)$.

6.2. The Brown-Peterson case. Let $B P$ be the $p$-local Brown-Peterson spectrum, realized as an $E_{4}$ symmetric ring spectrum [7], [8]. We could avoid using the $E_{4}$ structure on $B P$ by appealing to the symmetric ring spectrum map $M U \rightarrow B P$ of [6] and naturality, as in [14, 6.4], but this would make some arguments longer.

Recall [28, 4.1.12] the $\mathscr{A}_{*}$-comodule algebra isomorphism

$$
H_{*}(B P) \cong P\left(\bar{\xi}_{k} \mid k \geq 1\right) \text {. }
$$

Note that $H_{*}(B P)$ is concentrated in even degrees.

Lemma 6.7. There is an $\mathscr{A}_{*}$-comodule algebra isomorphism

$$
H_{*}(\mathrm{THH}(B P)) \cong H_{*}(B P) \otimes E\left(\sigma \bar{\xi}_{k} \mid k \geq 1\right) .
$$

The classes $\sigma \bar{\xi}_{k}$ are $\mathscr{A}_{*}$-comodule primitive, for all $k \geq 1$.

Proof. This is similar to the $M U$-case, see [3, 5.12].

Proposition 6.8. The homological Tate spectral sequence

$$
\widehat{E}_{*, *}^{2}(\mathrm{THH}(B P))=\widehat{H}^{-*}\left(C_{p} ; H_{*}(\mathrm{THH}(B P))\right) \Longrightarrow H_{*}^{c}\left(\mathrm{THH}(B P)^{t C_{p}}\right)
$$

collapses at the $\widehat{E}^{3}=\widehat{E}^{\infty}$-term, with

$$
\widehat{E}_{*, *}^{\infty}=\widehat{H}^{-*}\left(C_{p} ; \mathbb{F}_{p}\right) \otimes P\left(\bar{\xi}_{k}^{p} \mid k \geq 1\right) \otimes E\left(\bar{\xi}_{k}^{p-1} \sigma \bar{\xi}_{k} \mid k \geq 1\right) .
$$

Proof. This is similar to the $M U$-case. See also [14, 6.4].

Theorem 6.9. The map

$$
\gamma: \operatorname{THH}(B P) \rightarrow \operatorname{THH}(B P)^{t C_{p}}
$$

induces a complete $\mathscr{A}_{*}$-comodule algebra homomorphism

$$
\gamma_{*}: H_{*}(\mathrm{THH}(B P)) \longrightarrow H_{*}^{c}\left(\mathrm{THH}(B P)^{t C_{p}}\right)
$$

mapping

$$
\sigma \bar{\xi}_{k} \longmapsto t^{(p-1)\left(p^{k}-1\right)} \otimes \bar{\xi}_{k}^{p-1} \sigma \bar{\xi}_{k}
$$

modulo Tate filtration in the target, for each $k \geq 1$. 
Proof. This is similar to the $M U$-case, using the commutative diagram

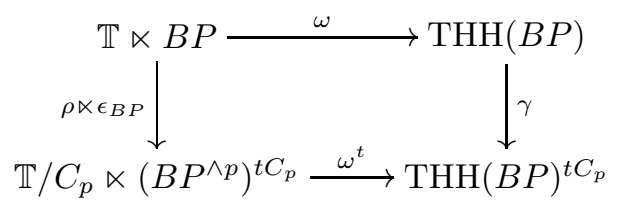

from Theorem 5.3, or naturality with respect to the symmetric ring spectrum map $M U \rightarrow B P$.

\section{The Segal Conjecture}

In this section we prove Theorem 2.2, which in turn implies Theorem 1.1. The main technical results are Propositions 7.2 and 7.3 .

Remark 7.1. The basic idea, in the case $B=M U$, is that the homological Tate spectral sequences

$$
{ }^{\prime} \widehat{E}_{*, *}^{2}=\widehat{H}^{-*}\left(C_{p} ; H_{*}(\mathrm{THH}(M U))\right) \Longrightarrow H_{*}^{c}\left(\mathrm{THH}(M U)^{t C_{p}}\right)
$$

and

have $\widehat{E}^{\infty}$-terms

$$
{ }^{\prime \prime} \widehat{E}_{*, *}^{2}=\widehat{H}^{-*}\left(C_{p} ; H_{*}\left(\mathrm{THH}(M U)^{\wedge p}\right)\right) \Longrightarrow R_{+}\left(H_{*}(\mathrm{THH}(M U))\right)
$$

$$
{ }^{\prime} \widehat{E}_{*, *}^{\infty}=E(u) \otimes P\left(t, t^{-1}\right) \otimes P\left(m_{\ell}^{p} \mid \ell \geq 1\right) \otimes E\left(m_{\ell}^{p-1} \sigma m_{\ell} \mid \ell \geq 1\right)
$$

and

$$
{ }^{\prime \prime} \widehat{E}_{*, *}^{\infty}=E(u) \otimes P\left(t, t^{-1}\right) \otimes P\left(m_{\ell} \otimes p \mid \ell \geq 1\right) \otimes E\left(\sigma m_{\ell} \otimes p \mid \ell \geq 1\right),
$$

that are isomorphic by way of a filtration-shifting isomorphism given by

$$
m_{\ell}^{p} \mapsto m_{\ell}^{\otimes p} \quad \text { and } \quad m_{\ell}^{p-1} \sigma m_{\ell} \mapsto t^{m} \otimes \sigma m_{\ell} \otimes p,
$$

where $m=(p-1) / 2$. The difficulty is to promote this isomorphism to an $\mathscr{A}_{*}$-comodule isomorphism of the abutments, respecting the linear topologies. Our approach is to compare the two Tate towers via a third tower, given by base changing the Tate tower for $M U^{\wedge p}$ along $\eta: M U \rightarrow \mathrm{THH}(M U)$. See diagram (7.3).

Consider any connective symmetric ring spectrum $B$. We have a commutative diagram

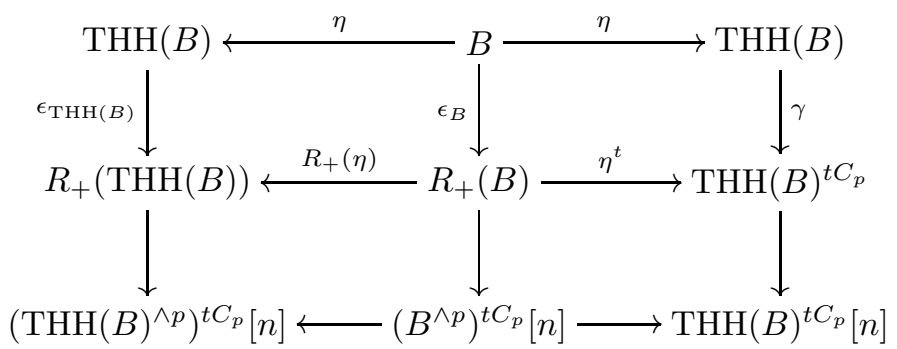

by Theorem 5.3 and naturality of $\epsilon$. We recall that $R_{+}(B)=\left(B^{\wedge p}\right)^{t C_{p}}$, while $\left(B^{\wedge p}\right)^{t C_{p}}[n]$ denotes the $n$ th term in the Tate tower (4.5), and similarly with $\operatorname{THH}(B)$ in place of $B$. We are principally concerned with the limit behavior as $n \rightarrow-\infty$.

Passing to continuous homology, we get a commutative diagram

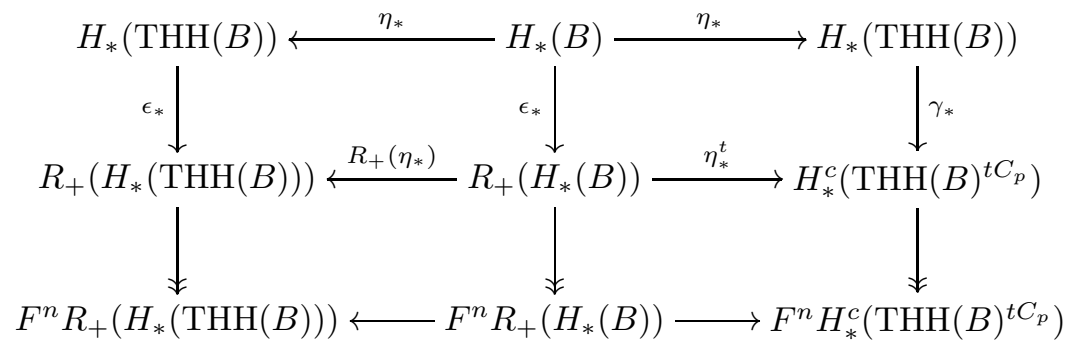

of complete $\mathscr{A}_{*}$-comodules. Here

$$
F^{n} R_{+}\left(H_{*}(B)\right)=\operatorname{im}\left(H_{*}^{c}\left(R_{+}(B)\right) \rightarrow H_{*}\left(\left(B^{\wedge p}\right)^{t C_{p}}[n]\right)\right)
$$


so that $R_{+}\left(H_{*}(B)\right)=\lim _{n} F^{n} R_{+}\left(H_{*}(B)\right)$, and similarly with $\operatorname{THH}(B)$ in place of $B$. We also use the notation

$$
F^{n} H_{*}^{c}\left(\mathrm{THH}(B)^{t C_{p}}\right)=\operatorname{im}\left(H_{*}^{c}\left(\operatorname{THH}(B)^{t C_{p}}\right) \rightarrow H_{*}\left(\operatorname{THH}(B)^{t C_{p}}[n]\right)\right)
$$

so that $H_{*}^{c}\left(\mathrm{THH}(B)^{t C_{p}}\right)=\lim _{n} F^{n} H_{*}^{c}\left(\mathrm{THH}(B)^{t C_{p}}\right)$.

Suppose now that $B$ is an $E_{2}$ symmetric ring spectrum, so that $\mathrm{THH}(B)$ is a ring spectrum [13] and the upper two rows of (17.1) form a diagram of ring spectra. Then the upper two rows of (7.2) form a diagram of complete $\mathscr{A}_{*}$-comodule algebras. Using the algebra structures, we get a commutative diagram given by the solid arrows below:

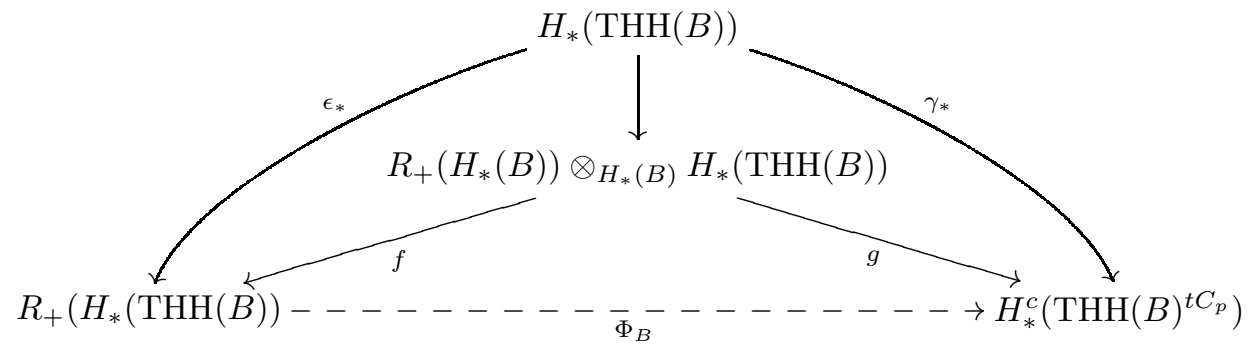

Here $f(\alpha \otimes \beta)=R_{+}\left(\eta_{*}\right)(\alpha) \cdot \epsilon_{*}(\beta)$ while $g(\alpha \otimes \beta)=\eta_{*}^{t}(\alpha) \cdot \gamma_{*}(\beta)$. We wish to construct a suitably structured isomorphism $\Phi_{B}: R_{+}\left(H_{*}(\mathrm{THH}(B))\right) \rightarrow H_{*}^{c}\left(\mathrm{THH}(B)^{t C_{p}}\right)$ making the whole diagram commute.

7.1. The case $B=M U$. In this case the central term in diagram (7.3) is

$$
R_{+}\left(H_{*}(M U)\right) \otimes_{H_{*}(M U)} H_{*}(\operatorname{THH}(M U)) \cong R_{+}\left(H_{*}(M U)\right) \otimes E\left(\sigma m_{\ell} \mid \ell \geq 1\right),
$$

since $H_{*}(\mathrm{THH}(M U)) \cong H_{*}(M U) \otimes E\left(\sigma m_{\ell} \mid \ell \geq 1\right)$.

By Lemma 6.2 each $\sigma m_{\ell}$ is $\mathscr{A}_{*}$-comodule primitive, so by [23, 3.2.1] we have $\epsilon_{*}\left(\sigma m_{\ell}\right)=1 \otimes \sigma m_{\ell}$ in $R_{+}\left(H_{*}(\mathrm{THH}(M U))\right.$ on the left hand side. It has Tate representative

$$
t^{m(2 \ell+1)} \otimes \sigma m_{\ell} \otimes p
$$

(up to a unit in $\mathbb{F}_{p}$ ) in Tate filtration $-(p-1)(2 \ell+1)$, by [23, 5.14], where $m=(p-1) / 2$. On the right hand side, we showed in Theorem 6.4 that $\gamma_{*}\left(\sigma m_{\ell}\right)$ has Tate representative

$$
t^{(p-1) \ell} \otimes m_{\ell}^{p-1} \sigma m_{\ell}
$$

(up to a sign) in Tate filtration $-2(p-1) \ell$.

Since both $\epsilon_{*}\left(\sigma m_{\ell}\right)$ and $\gamma_{*}\left(\sigma m_{\ell}\right)$ are in negative Tate filtration, we find that $f$ maps $\alpha \otimes \beta$, with $\alpha$ in Tate filtration $<n$ and $\beta \in E\left(\sigma m_{\ell} \mid \ell \geq 1\right)$, to a class in Tate filtration $<n$, and likewise for $g$. Hence we get a commutative diagram

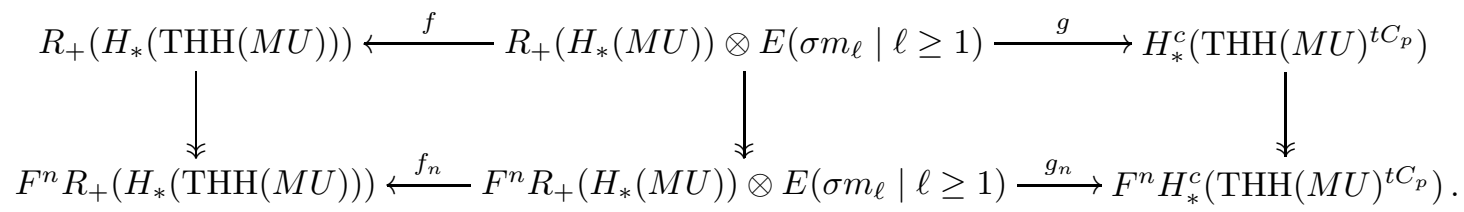

Each $f_{n}$ and $g_{n}$ is an $\mathscr{A}_{*}$-comodule homomorphism. Theorem 2.2 for $M U$ now follows from the proposition below by letting

$$
\Phi_{M U}=\widehat{g} \circ \widehat{f}^{-1}=\left(\lim _{n} g_{n}\right) \circ\left(\lim _{n} f_{n}\right)^{-1} .
$$

Recall the completed tensor product $\widehat{\otimes}$ from [23, §2.5].

Proposition 7.2. The homomorphisms

$$
f_{n}: F^{n} R_{+}\left(H_{*}(M U)\right) \otimes E\left(\sigma m_{\ell} \mid \ell \geq 1\right) \longrightarrow F^{n} R_{+}\left(H_{*}(\mathrm{THH}(M U))\right)
$$

and

$$
g_{n}: F^{n} R_{+}\left(H_{*}(M U)\right) \otimes E\left(\sigma m_{\ell} \mid \ell \geq 1\right) \longrightarrow F^{n} H_{*}^{c}\left(\mathrm{THH}(M U)^{t C_{p}}\right)
$$

define strict maps $\left\{f_{n}\right\}_{n}$ and $\left\{g_{n}\right\}_{n}$ of inverse systems of bounded below $\mathscr{A}_{*}$-comodules of finite type. These are pro-isomorphisms, in each homological degree. Hence each of the limiting homomorphisms

$$
\widehat{f}=\lim _{n} f_{n}: R_{+}\left(H_{*}(M U)\right) \widehat{\otimes} E\left(\sigma m_{\ell} \mid \ell \geq 1\right) \longrightarrow R_{+}\left(H_{*}(\operatorname{THH}(M U))\right)
$$

and

$$
\widehat{g}=\lim _{n} g_{n}: R_{+}\left(H_{*}(M U)\right) \widehat{\otimes} E\left(\sigma m_{\ell} \mid \ell \geq 1\right) \longrightarrow H_{*}^{c}\left(\mathrm{THH}(M U)^{t C_{p}}\right)
$$


is a continuous isomorphism of complete $\mathscr{A}_{*}$-comodules, with continuous inverse.

Proof. We begin with the proof for $\widehat{f}$. The part of the Tate spectral sequence $\widehat{E}^{\infty}$-term

$$
\begin{aligned}
\widehat{E}_{*, *}^{\infty} & =E(u) \otimes P\left(t, t^{-1}\right) \otimes P\left(m_{\ell} \otimes p\right. \\
& \Longrightarrow R_{+}\left(H_{*}(M U)\right)
\end{aligned}
$$

in bidegrees $(s, t)$ with Tate filtration $s \geq n$ is the associated graded of the preferred filtration of $F^{n} R_{+}\left(H_{*}(M U)\right)$. Hence the part of

$$
E(u) \otimes P\left(t, t^{-1}\right) \otimes P\left(m_{\ell} \otimes p \mid \ell \geq 1\right) \otimes E\left(\sigma m_{\ell} \mid \ell \geq 1\right)
$$

in Tate filtration $s \geq n$ is the associated graded of the filtration of $F^{n} R_{+}\left(H_{*}(M U)\right) \otimes E\left(\sigma m_{\ell} \mid \ell \geq 1\right)$. Similarly the part of the Tate spectral sequence $\widehat{E}^{\infty}$-term

$$
\begin{aligned}
\widehat{E}_{*, *}^{\infty} & =E(u) \otimes P\left(t, t^{-1}\right) \otimes P\left(m_{\ell}{ }^{\otimes p} \mid \ell \geq 1\right) \otimes E\left(\sigma m_{\ell}{ }^{\otimes p} \mid \ell \geq 1\right) \\
& \Longrightarrow R_{+}\left(H_{*}(\operatorname{THH}(M U))\right)
\end{aligned}
$$

in Tate filtration $s \geq n$ is the associated graded of the filtration of $F^{n} R_{+}\left(H_{*}(\mathrm{THH}(M U))\right)$.

The $\mathscr{A}_{*}$-comodule homomorphism $f_{n}$ is the identity on $F^{n} R_{+}\left(H_{*}(M U)\right)$, and takes $\sigma m_{\ell}$ to $\epsilon_{*}\left(\sigma m_{\ell}\right)$ represented by a unit times $t^{m(2 \ell+1)} \otimes \sigma m_{\ell}{ }^{\otimes p}$ modulo filtration. Notice that $t^{m(2 \ell+1)} \otimes \sigma m_{\ell}{ }^{\otimes p}$ lies in bidegree $(-(p-1)(2 \ell+1), p(2 \ell+1))$, on the line of slope $-p /(p-1)$ through the origin in the $(s, t)$-plane.

Now restrict attention to one total degree $d$, indicated by subscripts. We thus have compatible $\mathbb{F}_{p^{-}}$ linear homomorphisms

$$
f_{n, d}:\left[F^{n} R_{+}\left(H_{*}(M U)\right) \otimes E\left(\sigma m_{\ell} \mid \ell \geq 1\right)\right]_{d} \longrightarrow F^{n} R_{+}\left(H_{*}(\mathrm{THH}(M U))\right)_{d}
$$

for all integers $n$. To provide a pro-inverse, we shall define compatible $\mathbb{F}_{p}$-linear homomorphisms

$$
\phi_{n, d}: F^{N} R_{+}\left(H_{*}(\mathrm{THH}(M U))\right)_{d} \longrightarrow\left[F^{n} R_{+}\left(H_{*}(M U)\right) \otimes E\left(\sigma m_{\ell} \mid \ell \geq 1\right)\right]_{d}
$$

with $N=N(n, d)=p(n-d)+d$, for all integers $n$. Write the source of the inclusion

$$
\left[R_{+}\left(H_{*}(M U)\right) \otimes E\left(\epsilon_{*}\left(\sigma m_{\ell}\right) \mid \ell \geq 1\right)\right]_{d} \longrightarrow R_{+}\left(H_{*}(\mathrm{THH}(M U))\right)_{d}
$$

as a direct sum

$$
\bigoplus_{L}\left[R_{+}\left(H_{*}(M U)\right) \otimes \mathbb{F}_{p}\left\{\epsilon_{L}\right\}\right]_{d}
$$

with $L$ ranging over the strictly increasing sequences $L=\left(\ell_{1}<\cdots<\ell_{r}\right)$ of natural numbers, of length $r \geq 0$. Here

$$
\epsilon_{L}=\epsilon_{*}\left(\sigma m_{\ell_{1}}\right) \cdot \ldots \cdot \epsilon_{*}\left(\sigma m_{\ell_{r}}\right)
$$

has bidegree $\left(s_{L}, t_{L}\right)=(-(p-1)(2|L|+r), p(2|L|+r))$, where $|L|=\ell_{1}+\cdots+\ell_{r}$. The inclusion descends to an isomorphism

$$
\bigoplus_{L}\left[F^{N-s_{L}} R_{+}\left(H_{*}(M U)\right) \otimes \mathbb{F}_{p}\left\{\epsilon_{L}\right\}\right]_{d} \stackrel{\cong}{\longrightarrow} F^{N} R_{+}\left(H_{*}(\mathrm{THH}(M U))\right)_{d},
$$

as can be seen from the $\widehat{E}^{\infty}$-terms. Since $F^{N-s_{L}} R_{+}\left(H_{*}(M U)\right)$ is concentrated in total degrees $\geq N-s_{L}$, only the summands with $\left(N-s_{L}\right)+\left(s_{L}+t_{L}\right) \leq d$ are nonzero, and this is equivalent to $2|L|+r \leq d-n$. This inequality, in turn, implies that $N-s_{L} \leq n$. Hence we have homomorphisms

$$
\begin{aligned}
{\left[F^{N-s_{L}} R_{+}\left(H_{*}(M U)\right) \otimes \mathbb{F}_{p}\left\{\epsilon_{L}\right\}\right]_{d} } & \mapsto\left[F^{N-s_{L}} R_{+}\left(H_{*}(M U)\right) \otimes E\left(\sigma m_{\ell} \mid \ell \geq 1\right)\right]_{d} \\
& \rightarrow\left[F^{n} R_{+}\left(H_{*}(M U)\right) \otimes E\left(\sigma m_{\ell} \mid \ell \geq 1\right)\right]_{d}
\end{aligned}
$$

taking $\epsilon_{L}$ to $\sigma m_{\ell_{1}} \cdot \ldots \cdot \sigma m_{\ell_{r}}$. Taking the direct sum over $L$, and factoring through the isomorphism displayed above, we get the desired homomorphism $\phi_{n, d}$.

For varying $n$, the collection $\left\{\phi_{n, d}\right\}_{n}$ defines a pro-map, such that $f_{n, d} \circ \phi_{n, d}$ is equal to the structural surjection

$$
F^{N} R_{+}\left(H_{*}(\mathrm{THH}(M U))\right)_{d} \rightarrow F^{n} R_{+}\left(H_{*}(\mathrm{THH}(M U))\right)_{d},
$$

and $\phi_{n, d} \circ f_{N, d}$ is equal to the structural surjection

$$
\left[F^{N} R_{+}\left(H_{*}(M U)\right) \otimes E\left(\sigma m_{\ell} \mid \ell \geq 1\right)\right]_{d} \rightarrow\left[F^{n} R_{+}\left(H_{*}(M U)\right) \otimes E\left(\sigma m_{\ell} \mid \ell \geq 1\right)\right]_{d} .
$$

Hence $\left\{f_{n, d}\right\}_{n}$ is a pro-isomorphism, with pro-inverse $\left\{\phi_{n, d}\right\}_{n}$, in each total degree $d$. 
The proof for $\widehat{g}$ relies on similar filtration shift estimates. The associated graded of the filtration of $F^{n} R_{+}\left(H_{*}(M U)\right) \otimes E\left(\sigma m_{\ell} \mid \ell \geq 1\right)$ was discussed in the first part of the proof. The part of the Tate spectral sequence $\widehat{E}^{\infty}$-term

$$
\begin{aligned}
\widehat{E}_{*, *}^{\infty} & =E(u) \otimes P\left(t, t^{-1}\right) \otimes P\left(m_{\ell}^{p} \mid \ell \geq 1\right) \otimes E\left(m_{\ell}^{p-1} \sigma m_{\ell} \mid \ell \geq 1\right) \\
& \Longrightarrow H_{*}^{c}\left(\operatorname{THH}(M U)^{t C_{p}}\right)
\end{aligned}
$$

in Tate filtration $s \geq n$ is the associated graded of the filtration of $F^{n} H_{*}^{c}\left(\mathrm{THH}(M U)^{t C_{p}}\right)$.

The $\mathscr{A}_{*}$-comodule homomorphism $g_{n}$ identifies $F^{n} R_{+}\left(H_{*}(M U)\right)$ with the Tate filtration $s \geq n$ part of $E(u) \otimes P\left(t, t^{-1}\right) \otimes P\left(m_{\ell}^{p} \mid \ell \geq 1\right)$, taking $m_{\ell}^{\otimes p}$ to $m_{\ell}^{p}$. Furthermore, it takes $\sigma m_{\ell}$ to $\gamma_{*}\left(\sigma m_{\ell}\right)$, represented by a sign times $t^{(p-1) \ell} \otimes m_{\ell}^{p-1} \sigma m_{\ell}$ modulo filtration. Here $t^{(p-1) \ell} \otimes m_{\ell}^{p-1} \sigma m_{\ell}$ lies in bidegree $(-2(p-1) \ell, 2 p \ell+1)$, on the line of slope $-p /(p-1)$ through the point $(s, t)=(0,1)$.

In total degree $d$ we have the strict pro-map $\left\{g_{n, d}\right\}_{n}$, with components

$$
g_{n, d}:\left[F^{n} R_{+}\left(H_{*}(M U)\right) \otimes E\left(\sigma m_{\ell} \mid \ell \geq 1\right)\right]_{d} \longrightarrow F^{n} H_{*}^{c}\left(\mathrm{THH}(M U)^{t C_{p}}\right)_{d} .
$$

We define an $\mathbb{F}_{p}$-linear pro-inverse $\left\{\psi_{n, d}\right\}_{n}$, with components

$$
\psi_{n, d}: F^{N} H_{*}^{c}\left(\mathrm{THH}(M U)^{t C_{p}}\right)_{d} \longrightarrow\left[F^{n} R_{+}\left(H_{*}(M U)\right) \otimes E\left(\sigma m_{\ell} \mid \ell \geq 1\right)\right]_{d} .
$$

Here $N=N(n, d)=p(n-d)+d$, as in the $\widehat{f}$-case.

Write the source of the inclusion

$$
\left[R_{+}\left(H_{*}(M U)\right) \otimes E\left(\gamma_{*}\left(\sigma m_{\ell}\right) \mid \ell \geq 1\right)\right]_{d} \longrightarrow H_{*}^{c}\left(\mathrm{THH}(M U)^{t C_{p}}\right)_{d}
$$

as a direct sum

$$
\bigoplus_{L}\left[R_{+}\left(H_{*}(M U)\right) \otimes \mathbb{F}_{p}\left\{\gamma_{L}\right\}\right]_{d}
$$

with $L=\left(\ell_{1}<\cdots<\ell_{r}\right)$ as above and

$$
\gamma_{L}=\gamma_{*}\left(\sigma m_{\ell_{1}}\right) \cdot \ldots \cdot \gamma_{*}\left(\sigma m_{\ell_{r}}\right)
$$

in bidegree $\left(s_{L}^{\prime}, t_{L}^{\prime}\right)=(-2(p-1)|L|, 2 p|L|+r)$. The inclusion descends to an isomorphism

$$
\bigoplus_{L}\left[F^{N-s_{L}^{\prime}} R_{+}\left(H_{*}(M U)\right) \otimes \mathbb{F}_{p}\left\{\gamma_{L}\right\}\right]_{d} \cong F^{N} H_{*}^{c}\left(\operatorname{THH}(M U)^{t C_{p}}\right)_{d} .
$$

Only the summands with $\left(N-s_{L}^{\prime}\right)+\left(s_{L}^{\prime}+t_{L}^{\prime}\right) \leq d$ are nonzero, and this implies $2|L| \leq d-n$ since $r \geq 0$. This, in turn, implies $N-s_{L}^{\prime} \leq n$. Hence we have homomorphisms

$$
\begin{aligned}
{\left[F^{N-s_{L}^{\prime}} R_{+}\left(H_{*}(M U)\right) \otimes \mathbb{F}_{p}\left\{\gamma_{L}\right\}\right]_{d} } & \longmapsto\left[F^{N-s_{L}^{\prime}} R_{+}\left(H_{*}(M U)\right) \otimes E\left(\sigma m_{\ell} \mid \ell \geq 1\right)\right]_{d} \\
& \rightarrow\left[F^{n} R_{+}\left(H_{*}(M U)\right) \otimes E\left(\sigma m_{\ell} \mid \ell \geq 1\right)\right]_{d}
\end{aligned}
$$

taking $\gamma_{L}$ to $\sigma m_{\ell_{1}} \cdot \ldots \cdot \sigma m_{\ell_{r}}$. Summing over $L$, and using the isomorphism above, we get the required homomorphism $\psi_{n, d}$.

The collection $\left\{\psi_{n, d}\right\}_{n}$ defines a pro-map, such that $g_{n, d} \circ \psi_{n, d}$ is equal to the structural surjection

$$
F^{N} H_{*}^{c}\left(\mathrm{THH}(M U)^{t C_{p}}\right)_{d} \rightarrow F^{n} H_{*}^{c}\left(\mathrm{THH}(M U)^{t C_{p}}\right)_{d},
$$

and $\psi_{n, d} \circ g_{N, d}$ is equal to the structural surjection

$$
\left[F^{N} R_{+}\left(H_{*}(M U)\right) \otimes E\left(\sigma m_{\ell} \mid \ell \geq 1\right)\right]_{d} \rightarrow\left[F^{n} R_{+}\left(H_{*}(M U)\right) \otimes E\left(\sigma m_{\ell} \mid \ell \geq 1\right)\right]_{d} .
$$

Hence $\left\{g_{n, d}\right\}_{n}$ is a pro-isomorphism.

7.2. The case $B=B P$. In this case the central term in diagram (7.3) is

$$
R_{+}\left(H_{*}(B P)\right) \otimes_{H_{*}(B P)} H_{*}(\mathrm{THH}(B P)) \cong R_{+}\left(H_{*}(B P)\right) \otimes E\left(\sigma \bar{\xi}_{k} \mid k \geq 1\right) .
$$

By Lemma 6.7 each $\sigma \bar{\xi}_{k}$ is $\mathscr{A}_{*}$-comodule primitive, so by [23, 3.2.1] we have $\epsilon_{*}\left(\sigma \bar{\xi}_{k}\right)=1 \otimes \sigma \bar{\xi}_{k}$ in $R_{+}\left(H_{*}(\mathrm{THH}(B P))\right.$. It has Tate representative

$$
t^{m\left(2 p^{k}-1\right)} \otimes \sigma \bar{\xi}_{k} \otimes p
$$

in Tate filtration $-(p-1)\left(2 p^{k}-1\right)$, by [23, 5.14]. We showed in Theorem 6.9 that $\gamma_{*}\left(\sigma \bar{\xi}_{k}\right)$ has Tate representative

$$
t^{(p-1)\left(p^{k}-1\right)} \otimes \bar{\xi}_{k}^{p-1} \sigma \bar{\xi}_{k}
$$


in Tate filtration $-2(p-1)\left(p^{k}-1\right)$. Since both images are in negative Tate filtration, we get a commutative diagram

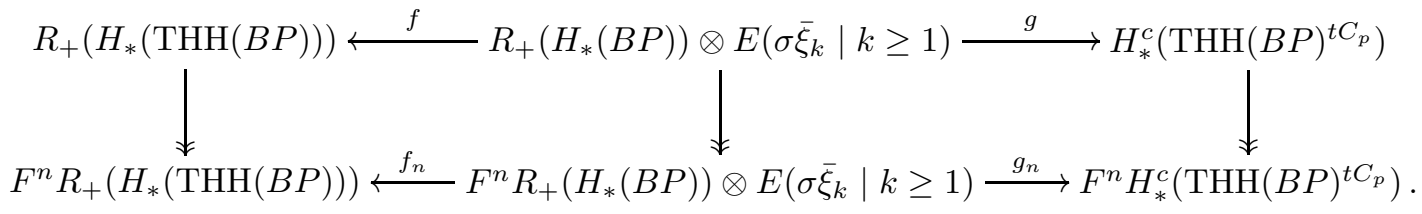

Theorem 2.2 for BP now follows from the proposition below by letting

$$
\Phi_{B P}=\widehat{g} \circ \widehat{f}^{-1}=\left(\lim _{n} g_{n}\right) \circ\left(\lim _{n} f_{n}\right)^{-1} .
$$

Proposition 7.3. The homomorphisms

$$
f_{n}: F^{n} R_{+}\left(H_{*}(B P)\right) \otimes E\left(\sigma \bar{\xi}_{k} \mid k \geq 1\right) \longrightarrow F^{n} R_{+}\left(H_{*}(\mathrm{THH}(B P))\right)
$$

and

$$
g_{n}: F^{n} R_{+}\left(H_{*}(B P)\right) \otimes E\left(\sigma \bar{\xi}_{k} \mid k \geq 1\right) \longrightarrow F^{n} H_{*}^{c}\left(\mathrm{THH}(B P)^{t C_{p}}\right)
$$

define strict maps $\left\{f_{n}\right\}_{n}$ and $\left\{g_{n}\right\}_{n}$ of inverse systems of bounded below $\mathscr{A}_{*}$-comodules of finite type. These are pro-isomorphisms, in each homological degree. Hence each of the limiting homomorphisms

$$
\widehat{f}=\lim _{n} f_{n}: R_{+}\left(H_{*}(B P)\right) \widehat{\otimes} E\left(\sigma \bar{\xi}_{k} \mid k \geq 1\right) \longrightarrow R_{+}\left(H_{*}(\mathrm{THH}(B P))\right)
$$

and

$$
\widehat{g}=\lim _{n} g_{n}: R_{+}\left(H_{*}(B P)\right) \widehat{\otimes} E\left(\sigma \bar{\xi}_{k} \mid k \geq 1\right) \longrightarrow H_{*}^{c}\left(\operatorname{THH}(B P)^{t C_{p}}\right)
$$

is a continuous isomorphism of complete $\mathscr{A}_{*}$-comodules, with continuous inverse.

Proof. This is similar to the $M U$-case.

\section{REFERENCES}

[1] J. F. Adams, Stable homotopy and generalised homology, University of Chicago Press, Chicago, Ill., 1974. Chicago Lectures in Mathematics.

[2] J. F. Adams, J. H. Gunawardena, and H. Miller, The Segal conjecture for elementary abelian p-groups, Topology 24 (1985), no. 4, 435-460.

[3] V. Angeltveit and J. Rognes, Hopf algebra structure on topological Hochschild homology, Algebr. Geom. Topol. 5 (2005), 1223-1290.

[4] Ch. Ausoni and J. Rognes, Algebraic K-theory of topological K-theory, Acta Math. 188 (2002), no. 1, 1-39.

[5] - The chromatic red-shift in algebraic K-theory, Guido's Book of Conjectures, Monographie de L'Enseignement Mathématique, vol. 40, 2008, pp. 13-15.

[6] A. Baker and A. Jeanneret, Brave new Hopf algebroids and extensions of MU-algebras, Homology Homotopy Appl. 4 (2002), no. 1, 163-173.

[7] M. Basterra and M. A. Mandell, Homology of $E_{n}$ ring spectra and iterated THH, Algebra. Geom. Topol. 11 (2011), 939-981.

[8] The multiplication in BP (2011). arXiv:1101.0023 preprint.

[9] M. Bökstedt, Topological Hochschild homology (ca. 1986). Bielefeld preprint.

[10] M. Bökstedt, W. C. Hsiang, and I. Madsen, The cyclotomic trace and algebraic K-theory of spaces, Invent. Math. 111 (1993), no. 3, 465-539.

[11] M. Bökstedt and I. Madsen, Topological cyclic homology of the integers, Astérisque 226 (1994), 7-8, 57-143. K-theory (Strasbourg, 1992).

[12] M. Bökstedt, R. R. Bruner, S. Lunøe-Nielsen, and J. Rognes, On cyclic fixed points of spectra (2007). arXiv:0712.3476 preprint.

[13] M. Brun, Z. Fiedorowicz, and R. M. Vogt, On the multiplicative structure of topological Hochschild homology, Algebr. Geom. Topol. 7 (2007), 1633-1650.

[14] R. R. Bruner and J. Rognes, Differentials in the homological homotopy fixed point spectral sequence, Algebr. Geom. Topol. 5 (2005), 653-690.

[15] G. Carlsson, Equivariant stable homotopy and Segal's Burnside ring conjecture, Ann. of Math. (2) 120 (1984), no. 2, $189-224$.

[16] J. Caruso, J. P. May, and S. B. Priddy, The Segal conjecture for elementary abelian p-groups. II. p-adic completion in equivariant cohomology, Topology 26 (1987), no. 4, 413-433.

[17] J. P. C. Greenlees, Representing Tate cohomology of G-spaces, Proc. Edinburgh Math. Soc. (2) 30 (1987), no. 3, $435-443$.

[18] J. P. C. Greenlees and J. P. May, Generalized Tate cohomology, Mem. Amer. Math. Soc. 113 (1995), no. 543, viii+178.

[19] L. Hesselholt and I. Madsen, On the K-theory of finite algebras over Witt vectors of perfect fields, Topology 36 (1997), no. 1, 29-101.

[20] M. Hovey, B. Shipley, and J. Smith, Symmetric spectra, J. Amer. Math. Soc. 13 (2000), no. 1, 149-208. 
[21] L. G. Lewis Jr., J. P. May, and M. Steinberger, Equivariant stable homotopy theory, Lecture Notes in Mathematics, vol. 1213, Springer-Verlag, Berlin, 1986. With contributions by J. E. McClure.

[22] W. H. Lin, D. M. Davis, M. E. Mahowald, and J. F. Adams, Calculation of Lin's Ext groups, Math. Proc. Cambridge Philos. Soc. 87 (1980), no. 3, 459-469.

[23] S. Lunøe-Nielsen and J. Rognes, The topological Singer construction (2010). arXiv:1010.5633 preprint.

[24] J. P. May, $E_{\infty}$ ring spaces and $E_{\infty}$ ring spectra, Lecture Notes in Mathematics, Vol. 577, Springer-Verlag, Berlin, 1977. With contributions by Frank Quinn, Nigel Ray, and Jørgen Tornehave.

[25] J. E. McClure and R. E. Staffeldt, On the topological Hochschild homology of bu. I, Amer. J. Math. 115 (1993), no. 1, $1-45$.

[26] J. Milnor, The Steenrod algebra and its dual, Ann. of Math. (2) 67 (1958), 150-171.

[27] D. C. Ravenel, The Segal conjecture for cyclic groups and its consequences, Amer. J. Math. 106 (1984), no. 2, 415-446. With an appendix by Haynes R. Miller.

[28] Complex cobordism and stable homotopy groups of spheres, Pure and Applied Mathematics, vol. 121, Academic Press Inc., Orlando, FL, 1986.

[29] J. Rognes, Trace maps from the algebraic K-theory of the integers (after M. Bökstedt), J. Pure Appl. Algebra 125 (1998), no. 1-3, 277-286.

Department of Mathematics, University of Oslo, Norway

E-mail address: sverreln@math.uio.no

$U R L$ : http://folk.uio.no/sverreln

Department of Mathematics, University of Oslo, Norway

E-mail address: rognes@math.uio.no

$U R L:$ http://folk.uio.no/rognes 